\title{
On stability of difference schemes. Central schemes for hyperbolic conservation laws with source terms
}

\author{
V. S. Borisov * M. Mond ${ }^{\dagger}$
}

June 17, 2018

\begin{abstract}
The stability of nonlinear explicit difference schemes with not, in general, open domains of the scheme operators are studied. For the case of path-connected, bounded, and Lipschitz domains, we establish the notion that a multi-level nonlinear explicit scheme is stable iff (if and only if) the corresponding scheme in variations is stable. A new modification of the central Lax-Friedrichs ( $\mathrm{LxF}$ ) scheme is developed to be of the second order accuracy. The modified scheme is based on nonstaggered grids. A monotone piecewise cubic interpolation is used in the central scheme to give an accurate approximation for the model in question. The stability of the modified scheme is investigated. Some versions of the modified scheme are tested on several conservation laws, and the scheme is found to be accurate and robust. As applied to hyperbolic conservation laws with, in general, stiff source terms, it is constructed a second order nonstaggered central scheme based on operator-splitting techniques.
\end{abstract}

\section{Introduction}

We are mainly concerned with the stability of nonlinear explicit difference schemes arising, e.g., in numerical analysis of nonlinear PDE (partial differential equation, such as parabolic, hyperbolic, etc.) systems, generally, in multidimensional space. Stability is the central and the most pressing problem in any algorithm 48. Nowadays, there exists a few methods for stability analysis of some classes of difference schemes approximating PDE systems (see, e.g., [16, [17, 32, 35, 38, 48, and references therein). However, the problem of stability analysis for nonlinear schemes is still one of the most burning problems,

\footnotetext{
*The Pearlstone Center for Aeronautical Engineering Studies, Department of Mechanical Engineering, Ben-Gurion University of the Negev, Beer-Sheva, Israel. E-mail: viatslav@bgu.ac.il

${ }^{\dagger}$ The Pearlstone Center for Aeronautical Engineering Studies, Department of Mechanical Engineering, Ben-Gurion University of the Negev, Beer-Sheva, Israel. E-mail: mond@bgu.ac.il
} 
because of the absence of its complete solution [17. In particular, the vast majority of difference schemes, currently in use, have still not been analyzed [16, and, in general, no numerical method for non-linear systems of equations has been proven to be stable 32. A new approach to testing scheme stability has been suggested in [7] to prove convergence of non-linear schemes for systems of PDEs. It was demonstrated [7] that the notion of scheme in variations (or variational scheme [9], 10]) has much potential to be an effective tool for studying stability of nonlinear schemes. The scheme in variations for a difference scheme represents a tangent space at a point of the manifold associated to the difference scheme [7, 9]. Thus, the scheme in variations will always be linear and, hence, enables the investigation of the stability for nonlinear operators using, in general, an infinite family of linear patterns. It was established in 7 . the notion that the stability of a scheme in variations implies the stability of its original scheme, and that a nonlinear explicit scheme will be stable iff (if and only if) its scheme in variations will be stable. In this paper we consider difference schemes with not, in general, open domains of the scheme operators. For the case of internal path-connected, bounded, and Lipschitz domains, we establish the notion that a multi-level nonlinear explicit scheme is stable iff the corresponding scheme in variations is stable.

By way of illustration of the developed approach we are concerned with the stability analysis of central difference schemes for hyperbolic systems of conservation laws with source terms. Such systems are used to describe many physical problems of great practical importance in magneto-hydrodynamics, kinetic theory of rarefied gases, linear and nonlinear waves, viscoelasticity, multi-phase flows and phase transitions, shallow waters, etc. (see, e.g., 66, 12, 18, 24, [29, 32, 34, 37], 41], 42]). We will consider a system of hyperbolic conservation laws written as follows (e.g., [18, 32])

$$
\frac{\partial \mathbf{u}}{\partial t}+\sum_{j=1}^{N} \frac{\partial}{\partial x_{j}} \mathbf{f}_{j}(\mathbf{u})=\frac{1}{\tau} \mathbf{q}(\mathbf{u}), 0<t \leq T_{\max },\left.\mathbf{u}(\mathbf{x}, t)\right|_{t=0}=\mathbf{u}^{0}(\mathbf{x}),
$$

where the column-vector $\mathbf{x} \equiv\left\{x_{1}, x_{2}, \ldots, x_{N}\right\}^{T} \in \mathbb{R}^{N}, \mathbf{u}=\left\{u_{1}, u_{2}, \ldots, u_{M}\right\}^{T}$ is a vector-valued function from $\mathbb{R}^{N} \times[0,+\infty)$ into a subset $\Omega_{\mathbf{u}} \subset \mathbb{R}^{M}, \mathbf{f}_{j}(\mathbf{u})=$ $\left\{f_{1 j}(\mathbf{u}), f_{2 j}(\mathbf{u}), \ldots, f_{M j}(\mathbf{u})\right\}^{T}$ is a smooth function (flux-function) from $\Omega_{\mathbf{u}}$ into $\mathbb{R}^{M}, \mathbf{q}(\mathbf{u})=\left\{q_{1}(\mathbf{u}), q_{2}(\mathbf{u}), \ldots, q_{M}(\mathbf{u})\right\}^{T}$ denotes the source term, $\tau>0$ denotes the stiffness parameter, $\mathbf{u}^{0}(\mathbf{x})$ is of compact support. It is assumed that $\tau=$ const without loss of generality. It is also assumed that all eigenvalues, $\xi_{k}=\xi_{k}(\mathbf{u})$, of the Jacobian matrix $\mathbf{G}(=\partial \mathbf{q}(\mathbf{u}) / \partial \mathbf{u})$ have non-positive real parts, i.e.

$$
R_{e} \xi_{k}(\mathbf{u}) \leq 0, \quad \forall k, \quad \forall \mathbf{u} \in \Omega_{\mathbf{u}}
$$

In what follows $\|\mathbf{M}\|_{p}$ denotes the matrix norm of a matrix $\mathbf{M}$ induced by the vector norm $\|\mathbf{v}\|_{p}=\left(\sum_{i}\left|v_{i}\right|^{p}\right)^{1 / p}$, and $\|\mathbf{M}\|$ denotes the matrix norm induced by a prescribed vector norm. $\mathbb{R}$ denotes the field of real numbers. The transpose of a matrix (or vector) $\mathbf{S}$ is denoted by $\mathbf{S}^{T}$. The null element in any linear space, as well as the number zero, will be denoted by the same symbol 0 . 
Central schemes are attractive for various reasons: no Riemann solvers, characteristic decompositions, complicated flux splittings, etc., must be involved in construction of a central scheme (see, e.g., [5], 8], [23], [29], [30, [32, [39], 41], [43] and references therein). However, there is the need for staggered central schemes to alternate between two staggered grids, which can be cumbersome, e.g., near the boundaries [23]. Moreover, there is a risk for every central scheme to exhibit spurious solutions [8] in spite of sufficiently small CFL (CourantFriedrichs-Lewy 32]) number. To simplify implementation of central schemes in the case of complex geometries and boundary conditions, it was developed nonstaggered central schemes in 23. A new second-order modification of the staggered LxF scheme was developed in [8] to avoid the risk of spurious oscillations. It was demonstrated in 8 that the higher order versions of LxF scheme can produce spurious oscillations because of a negative numerical viscosity introduced to increase accuracy of the scheme up to $O\left(\Delta t+(\Delta x)^{2}\right)$. To reduce the risk of spurious solutions, it was suggested in 8 to introduce an additional non-negative numerical viscosity such that the scheme's order of accuracy becomes $O\left((\Delta t)^{2}+(\Delta x)^{2}\right)$. The developed central scheme was tested on several conservation laws taking a CFL number equal or close to unity, and the scheme was found to be accurate and robust. In this paper, we extend the second-order modification to a new nonstaggered central scheme. The stability of this scheme is proven in Section 3.3 on the basis of the approach developed in Section 2 as well as in [7, [8]. The scheme is tested on several conservation laws in Section 5.

A stable numerical scheme may yield spurious results when applied to a stiff hyperbolic system with relaxation (see, e.g., [1, 4], 6], [12, [13, 24, [44, 45]). It is significant that a numerical scheme for relaxation systems must possess a discrete analogy to the continuous asymptotic limit, because any scheme violating the correct asymptotic limit leads to spurious or poor solutions (see, e.g., 12, [24, 25], 37, [41]). Most methods for solving such systems can be described as operator splitting ones, 13, or methods of fractional steps, 6. After operator splitting, one solves the advection homogeneous system, and then the ordinary differential equations associated with the source terms. We are mainly concerned with such an approach in Section 4 .

\section{Stability of nonlinear explicit schemes}

We consider the following $(q+1)$-level difference scheme:

$$
\begin{aligned}
\mathbf{w}_{i}^{n+1}= & \mathbf{G}_{i}^{n}\left(\mathbf{w}_{1}^{n}, \mathbf{w}_{1}^{n-1}, \ldots, \mathbf{w}_{1}^{n-q+1}, \mathbf{w}_{2}^{n}, \mathbf{w}_{2}^{n-1}, \ldots, \mathbf{w}_{2}^{n-q+1}, \ldots,\right. \\
& \left.\mathbf{w}_{I}^{n}, \mathbf{w}_{I}^{n-1}, \ldots, \mathbf{w}_{I}^{n-q+1}\right), \quad \mathbf{G}_{i}^{n}: \Omega_{n} \subseteq \mathbb{R}^{N} \rightarrow \mathbb{R}^{N_{0}},
\end{aligned}
$$

where $q, N_{0}, I \geq 1$ are finite integer constants, $N=q N_{0} I, \mathbf{w}_{i}^{k} \in \mathbb{R}^{N_{0}}$ denotes a vector-valued grid function, $i \in \omega_{1}$ denotes a node of the grid $\omega_{1} \equiv\{1,2, \ldots, I\}$, $k \in \omega_{2}$ denotes a node (time level) of the grid $\omega_{2} \equiv\{0,1, \ldots, M\}, M \geq q$ is a finite integer constant. $\mathbf{G}_{i}^{n}=\left\{G_{i, 1}^{n}, G_{i, 2}^{n}, \ldots, G_{i, N_{0}}^{n}\right\}^{T}$ denotes the vector-valued 
function with the domain and range belonging to $\mathbb{R}^{N}$ and $\mathbb{R}^{N_{0}}$, respectively. We emphasize that the domain, $\Omega_{n}$, of the function $\mathbf{G}_{i}^{n}$ is the set of argument values, which need not be open in $\mathbb{R}^{N}$. We assume that $n$ in (3) denotes the time level, $t_{n}(=n \Delta t)$. Thus, the time increment will be represented by $\Delta t=t_{\max } / M=$ const, where $t_{\max }$ denotes some finite time over which we wish to compute. Using the notation

$$
\begin{gathered}
\mathbf{H}_{i}^{n}=\left\{\left(\mathbf{G}_{i}^{n}\right)^{T},\left(\mathbf{w}_{i}^{n}\right)^{T},\left(\mathbf{w}_{i}^{n-1}\right)^{T}, \ldots,\left(\mathbf{w}_{i}^{n-q+2}\right)^{T}\right\}^{T}, \\
\mathbf{v}_{i}^{n}=\left\{\left(\mathbf{w}_{i}^{n}\right)^{T},\left(\mathbf{w}_{i}^{n-1}\right)^{T}, \ldots,\left(\mathbf{w}_{i}^{n-q+1}\right)^{T}\right\}^{T}, \quad i \in \omega_{1}, n \in \omega_{2},
\end{gathered}
$$

we reduce (see, e.g., [15, 47]) the multi-level scheme, (3), to the following two-level scheme:

$$
\mathbf{v}_{i}^{n+1}=\mathbf{H}_{i}^{n}\left(\mathbf{v}_{1}^{n}, \mathbf{v}_{2}^{n}, \ldots, \mathbf{v}_{I}^{n}\right), \mathbf{H}_{i}^{n}: \Omega_{n} \subseteq \mathbb{R}^{N} \rightarrow \mathbb{R}^{q N_{0}}, i \in \omega_{1}, n, n+1 \in \omega_{2}
$$

If we define

$$
\mathbf{v}^{n}=\left\{\left(\mathbf{v}_{1}^{n}\right)^{T},\left(\mathbf{v}_{2}^{n}\right)^{T}, \ldots,\left(\mathbf{v}_{I}^{n}\right)^{T}\right\}^{T}, \mathbf{H}^{n}=\left\{\left(\mathbf{H}_{1}^{n}\right)^{T},\left(\mathbf{H}_{2}^{n}\right)^{T}, \ldots,\left(\mathbf{H}_{I}^{n}\right)^{T}\right\}^{T}
$$

then the scheme (5) becomes

$$
\mathbf{v}^{n+1}=\mathbf{H}^{n}\left(\mathbf{v}^{n}\right), \quad \mathbf{H}^{n}: \Omega_{n} \subseteq \mathbb{R}^{N} \rightarrow \mathbb{R}^{N}, \quad n, n+1 \in \omega_{2} \equiv\{0,1, \ldots, M\}
$$

As usual (e.g., [40, p. 62]), for mappings $\mathbf{f}: \Omega_{f} \subseteq \mathbb{R}^{N} \rightarrow \mathbb{R}^{N}$ and $\mathbf{g}: \Omega_{g} \subseteq$ $\mathbb{R}^{N} \rightarrow \mathbb{R}^{N}$, the composite mapping $\mathbf{h}=\mathbf{g} \circ \mathbf{f}$ is defined by $\mathbf{h}(\mathbf{v})=\mathbf{g}(\mathbf{f}(\mathbf{v}))$ for all $\mathbf{v} \in \Omega_{h}=\left\{\mathbf{v} \in \Omega_{f} \mid \mathbf{f}(\mathbf{v}) \in \Omega_{g}\right\}$. Using the composite mapping approach, we rewrite Scheme (7) to read

$$
\mathbf{y}=\mathbf{F}(\mathbf{x}), \quad \mathbf{F}: \Omega_{F} \subseteq \mathbb{R}^{N} \rightarrow \mathbb{R}^{N}
$$

where the following notation is used: $\mathbf{x}=\mathbf{v}^{0}, \mathbf{y}=\mathbf{v}^{M}, \mathbf{F}=\mathbf{H}^{M-1} \circ \mathbf{H}^{M-2} \circ \ldots \circ$ $\mathbf{H}^{0}, \Omega_{F}=\left\{\mathbf{v}^{0} \in \Omega_{0}\left|\mathbf{v}^{1}=\mathbf{H}^{0}\left(\mathbf{v}^{0}\right) \in \Omega_{1}\right| \ldots \mid \mathbf{v}^{M-1}=\mathbf{H}^{M-2}\left(\mathbf{v}^{M-2}\right) \in \Omega_{M-1}\right\}$. Notice, $\mathbf{F}$ in (8) depends also on scheme parameters (e.g., space and time increments), however, this dependence is usually not included in the notation. Let the scheme parameters (including time increments) be represented by a vector $\mathbf{s}$ belonging to some normed space with the norm $|\mathbf{s}|$.

Let us remind the well-known definition of stability of an explicit scheme (see, e.g., [16, [18, 32, 47], 48, 49]).

Definition 1 Scheme (8) is said to be stable if there exist positive constants $s_{0}$ and $C$ such that for all $\mathbf{x}, \mathbf{x}_{*} \in \Omega_{F}$ the following inequality is valid

$$
\left\|\mathbf{F}\left(\mathbf{x}_{*}\right)-\mathbf{F}(\mathbf{x})\right\| \leq C\left\|\mathbf{x}_{*}-\mathbf{x}\right\|, \quad \forall \mathbf{s}:|\mathbf{s}| \leq s_{0}
$$


Thus, scheme (8) and, hence, scheme (3) will be stable iff the function $\mathbf{F}$ will be Lipschitz for a constant $C$. Notice that this well-known definition of stability may lead to wrong conclusion. Actually, let us consider the "slit plane" [21] in polar coordinates $(r, \theta)$

$$
\Omega_{F}=\{(r, \theta) \mid 0<r<\infty,-\pi<\theta<\pi\} \subset \mathbb{R}^{2},
$$

and the vector-valued function $\mathbf{F}=\left\{F_{1}(r, \theta), F_{2}(r, \theta)\right\}^{T}$ such that [21]

$$
F_{1}=r, F_{2}=\theta / 2 \text {. }
$$

We take $(r, \theta)_{*}=\left(r_{0},-\pi+\varepsilon\right),(r, \theta)=\left(r_{0}, \pi-\varepsilon\right)$, and $r_{0}=$ const. Considering the Euclidean distance between these two points of the plane with Cartesian coordinates $\mathbf{x}_{*} \equiv\left(-r_{0} \cos \varepsilon,-r_{0} \sin \varepsilon\right)$ and $\mathbf{x} \equiv\left(-r_{0} \cos \varepsilon, r_{0} \sin \varepsilon\right)$ we find that the inequality (9) takes the form: $\cos (\varepsilon / 2) \leq C \sin \varepsilon$. Obviously the mapping (11) is not Lipschitz, since $C$ in (9) tends to infinity as $\varepsilon \rightarrow 0$. Therefore, we have to conclude, in view of the above definition, that the scheme (11) is not stable, even though for every point $(r, \theta)$ in $\Omega_{F}$, (10), there exists a neighborhood of $(r, \theta)$ such that the function $\mathbf{F}$, (11), restricted to the neighborhood is Lipschitz for $C=1$, i.e. the function $\mathbf{F}$ is locally Lipschitz. Further, $\mathbf{F}$ is the nonstretching mapping of the "slit plane" (10) into the right semi-plane. Hence, the preceding definition of stability, i.e. Definition 1 needs to be slightly improved. Such a problem has been considered in [7] under the assumption that the domain $\Omega_{F}$ of the vector-valued function $\mathbf{F}$, (8), is open in $\mathbb{R}^{N}$. We will assume that the Lebesgue-measurable domain $\Omega_{F}$ of the function $\mathbf{F}$, (8), is a not, in general, open subset of $\mathbb{R}^{N}$ with non-empty interior.

We shall start with some basic notions. A set $\Omega \subseteq \mathbb{R}^{N}$ is said to be internal path-connected (or path-connected, in the case that $\Omega$ is open) if every two points $\mathbf{x}, \mathbf{x}_{*} \in \Omega$ can be joined by a continuous curve $\gamma:[0,1] \subset \mathbb{R} \rightarrow \Omega$ of finite length, $L(\gamma)$, with int $(\gamma) \subseteq$ int $(\Omega)$. Here and in what follows int $(\Omega)$ denotes the interior of $\Omega \subseteq \mathbb{R}^{N}$ and $\operatorname{int}(\gamma) \equiv \gamma \backslash(\gamma(0) \cup \gamma(1))$ denotes the interior of the continuous curve $\gamma$. The intrinsic metric $\Lambda_{\Omega}$ on an internal path-connected set $\Omega$ is defined as

$$
\Lambda_{\Omega}\left(\mathbf{x}, \mathbf{x}_{*}\right)=\inf _{\gamma \subseteq \Omega} L(\gamma), \quad \mathbf{x}=\gamma(0), \mathbf{x}_{*}=\gamma(1), \mathbf{x}, \mathbf{x}_{*} \in \Omega .
$$

An open ball (of radius $r>0$ ) about $\mathbf{x} \in \mathbb{R}^{N}$ is denoted by $B(\mathbf{x}, r)$ (or just $B_{\mathbf{x}}$ ). A closed ball is denoted by $\bar{B}(\mathbf{x}, r)$ (or just $\bar{B}_{\mathbf{x}}$ ). Given points $\mathbf{x}, \mathbf{y} \in \mathbb{R}^{N}$, given an open ball $B\left(\mathbf{x}, r_{\mathbf{x}}\right)$, and given an open ball $B\left(\mathbf{y}, r_{\mathbf{y}}\right)$ such that $\mathbf{x} \notin B_{\mathbf{y}}$, the convex open set $Q_{\mathbf{x}}=\left\{\mathbf{z} \mid \mathbf{x}+\lambda(\mathbf{z}-\mathbf{x}), \lambda>0, \mathbf{z} \in B_{\mathbf{y}}\right\} \cap B_{\mathbf{x}}$ will be called a finite cone with vertex $\mathbf{x}$. A set $\Omega \subseteq \mathbb{R}^{N}$ is said to have the cone property if there exists a finite cone $Q_{*}$ such that each boundary point $\mathbf{x} \in \partial \Omega$ is the vertex of a finite cone $Q_{\mathrm{x}} \subseteq \operatorname{int}(\Omega)$ congruent to $Q_{*}$. Suppose that $\Omega \subseteq \mathbb{R}^{N}$ has the cone property, and let $K_{\mathbf{x}}$ denote the union of all finite cones with the vertex $\mathbf{x} \in \partial \Omega$, i.e. $K_{\mathbf{x}}=\cup Q_{\mathbf{x}}$. Then a function $\mathbf{F}: \Omega \subseteq \mathbb{R}^{N} \rightarrow \mathbb{R}^{N}$ will be called locally Lipschitz (or locally Lipschitz continuous) if for every $\mathbf{x} \in \Omega$ there exist $B_{\mathbf{x}}$ and a constant $c_{\mathbf{x}}>0$ such that $\|\mathbf{F}(\mathbf{x})-\mathbf{F}(\mathbf{y})\| \leq c_{\mathbf{x}}\|\mathbf{x}-\mathbf{y}\|$ for each 
$\mathbf{y} \in B_{\mathbf{x}}$ provided $\mathbf{x} \in \operatorname{int}(\Omega)$, or $\mathbf{y} \in B_{\mathbf{x}} \cap K_{\mathbf{x}}$ otherwise. $\Omega \subset \mathbb{R}^{N}$ is Lipschitz (has a Lipschitz boundary), if for each point $\mathbf{x} \in \partial \Omega$ there exists an open ball $B_{\mathbf{x}}$ such that $\partial \Omega \cap B_{\mathbf{x}}$ is the graph of a Lipschitz function and int $(\Omega)$ lies on one side of its boundary (for more details see, e.g., [11, p. 149], [31, p. 354]). Let us note that $\Omega \subset \mathbb{R}^{N}$ satisfies the cone property if it has a Lipschitz boundary (see, e.g., [11, p. 151], 31, p. 355]), but not vice-versa as the example (10) shows. $W^{1, \infty}(\Omega)$ denotes the Sobolev space (see Definition 5 in [11, p. 28], see also [21] and [31]), i.e., the space consisting of all bounded functions on open $\Omega \subset \mathbb{R}^{N}$ whose distributional derivatives are bounded functions as well. Let $\mathbf{F} \equiv\left\{F_{1}\right.$, $\left.F_{2}, \ldots, F_{N}\right\}^{T}$ in (8), let $\nabla F_{i} \equiv\left\{\partial_{1} F_{i}, \ldots, \partial_{N} F_{i}\right\}$ denote the distributional gradient of $F_{i}$, and let $\delta \mathbf{F}, \delta \mathbf{x} \in \mathbb{R}^{N}$ denote variations. The following equality

$$
\delta \mathbf{F}=\mathbf{F}^{\prime} \cdot \delta \mathbf{x}, \quad \mathbf{F}^{\prime} \equiv\left\{\left(\nabla F_{1}\right)^{T},\left(\nabla F_{2}\right)^{T}, \ldots,\left(\nabla F_{N}\right)^{T}\right\}^{T},
$$

will be viewed as the scheme in variations for (8). The matrix representation of $\mathbf{F}^{\prime}$ in (13) is given by the following Jacobian matrix [40]: $\mathbf{F}^{\prime}=\left\{\partial F_{i} / \partial x_{j}\right\}$, $i, j=1,2, \ldots, N$.

Definition 2 Let $\Omega_{F}$ in (8) be internal path-connected. Scheme (8) is said to be stable if there exist positive constants $s_{0}$ and $C$ such that the following inequality holds

$$
\left\|\mathbf{F}\left(\mathbf{x}_{*}\right)-\mathbf{F}(\mathbf{x})\right\| \leq C \Lambda_{\Omega_{F}}\left(\mathbf{x}, \mathbf{x}_{*}\right), \quad \forall \mathbf{x}, \mathbf{x}_{*} \in \Omega_{F}, \quad \forall \mathbf{s}:|\mathbf{s}| \leq s_{0} .
$$

Notice that the scheme (11) is stable in terms of Definition 2 since the inequality (14) holds for $C=1$. It is significant that the domain $\Omega_{F}$, (10), of the function $\mathbf{F}$, (11), is open in $\mathbb{R}^{2}$. However, if the domain of the function $\mathbf{F}$, (11), is not open, then the situation can be in complete contrast to the previous one. Actually, let us add to $\Omega_{F}$, (10), the only boundary point $(1, \pi)$, i.e. let $\Omega_{F}^{*}=\Omega_{F} \cup(1, \pi)$ will be the domain of the function $\mathbf{F}^{*}=\mathbf{F}$, (11), if $(r, \theta) \in \Omega_{F}$ and $\mathbf{F}^{*}=(1, \pi / 2)$ if $(r, \theta)=(1, \pi)$. We take $(r, \theta)_{*}=(1,-\pi+\varepsilon),(r, \theta)=(1, \pi)$. Considering the Euclidean distance between these two points of the plane with Cartesian coordinates $\mathbf{x}_{*} \equiv\left(-\cos \varepsilon,-r_{0} \sin \varepsilon\right)$ and $\mathbf{x} \equiv(-1,0)$ we find that the inequality (14) takes the form: $\cos (\varepsilon / 4) \leq C \sin (\varepsilon / 2)$. Thus, the scheme (11), namely $\mathbf{F}: \Omega_{F}^{*} \subset \mathbb{R}^{2} \rightarrow \mathbb{R}^{2}$, is not stable, since $C$ in (14) tends to infinity as $\varepsilon \rightarrow 0$.

Lemma 3 Let the path-connected $\Omega_{F}$ of (8) be open in $\mathbb{R}^{N}$. Scheme (8) will be stable in terms of Definition $⿴$ : iff $\mathbf{F}$ in (8) will be locally Lipschitz for a common constant $C$, for all scheme parameters $\mathbf{s}$ such that $|\mathbf{s}| \leq s_{0}$.

Proof. Suppose Scheme (8) is stable, i.e. (14) is valid. Choose any point $\mathbf{x} \in \Omega_{F}$. Since $\Omega_{F}$ is open, there exists a radius $r$ such that $B(\mathbf{x}, r) \subset \Omega_{F}$. Choose any point $\mathbf{x}_{*} \in B(\mathbf{x}, r)$, and let $\gamma_{*}$ be the straight line segment joining the points $\mathbf{x}, \mathbf{x}_{*} \in B(\mathbf{x}, r)$. In view of (14), $\mathbf{F}$ in (8) will be locally Lipschitz for a common constant $C$, for all $\mathbf{s}:|\mathbf{s}| \leq s_{0}$, since $\Lambda_{\Omega_{F}}\left(\mathbf{x}, \mathbf{x}_{*}\right)=L\left(\gamma_{*}\right)=\left\|\mathbf{x}_{*}-\mathbf{x}\right\|$. 
Conversely, suppose that $\mathbf{F}$ in (8) is locally Lipschitz for a common constant $C$, for all $\mathbf{s}:|\mathbf{s}| \leq s_{0}$. Let some points $\mathbf{x}, \mathbf{x}_{*} \in \Omega_{F}$ be joined by a continuous curve $\gamma$. In view of (12), the curve $\gamma$ can be taken such that $L(\gamma) \leq \Lambda_{\Omega_{F}}\left(\mathbf{x}, \mathbf{x}_{*}\right)+$ $\varepsilon$ for an arbitrary $\varepsilon>0$. Given any point $\mathbf{z} \in \gamma$, there is a ball $B_{\mathbf{z}} \subset \Omega_{F}$ such that $\mathbf{F}$ restricted to $B_{\mathbf{z}}$ is Lipschitz for the common constant $C$ (locally Lipschitz continuity). The balls $\left\{B_{\mathbf{z}}\right\}$ form an open cover of $\gamma$. Since the mapping $\gamma:[0,1] \subset \mathbb{R} \rightarrow \mathbb{R}^{N}$ is continuous, the curve $\gamma$ is compact [28, p. 94]. Hence, by the compactness of $\gamma,\left\{B_{\mathbf{z}}\right\}$ has a finite subcover consisting of balls $B_{\mathbf{x}}=B_{\mathbf{z}_{1}}, B_{\mathbf{z}_{2}}, \ldots, B_{\mathbf{z}_{K}}=B_{\mathbf{x}_{*}}$. Since $\mathbf{F}$ is locally Lipschitz, we find

$$
\left\|\mathbf{F}\left(\mathbf{z}_{k+1}\right)-\mathbf{F}\left(\mathbf{z}_{k}\right)\right\| \leq C\left\|\mathbf{z}_{k+1}-\mathbf{z}_{k}\right\|, \quad k=1,2, \ldots K-1, \forall \mathbf{s}:|\mathbf{s}| \leq s_{0} .
$$

Then, by virtue of (15), we find

$$
\begin{gathered}
\left\|\mathbf{F}\left(\mathbf{x}_{*}\right)-\mathbf{F}(\mathbf{x})\right\|=\left\|\sum_{k}\left[\mathbf{F}\left(\mathbf{z}_{k+1}\right)-\mathbf{F}\left(\mathbf{z}_{k}\right)\right]\right\| \leq C \sum_{k}\left\|\mathbf{z}_{k+1}-\mathbf{z}_{k}\right\| \leq \\
C L(\gamma) \leq C \Lambda_{\Omega_{F}}\left(\mathbf{x}, \mathbf{x}_{*}\right)+\varepsilon C, \quad \forall \mathbf{s}:|\mathbf{s}| \leq s_{0} .
\end{gathered}
$$

By letting $\varepsilon \rightarrow 0$, we find that (14) holds.

Notice, in view of Lemma3, the scheme (11) is stable in terms of Definition2 since the domain $\Omega_{F}$, (10), is open in $\mathbb{R}^{2}$ and path-connected, and the function $\mathbf{F}$, (11), is locally Lipschitz for the common constant $C=1$.

Theorem 4 Let the internal path-connected $\Omega_{F}$ of (8) have the cone property, then the scheme (8) will be stable in terms of Definition 2 iff $\mathbf{F}$ in (8) will be locally Lipschitz for a common constant $C$, for all $\mathbf{s}$ such that $|\mathbf{s}| \leq s_{0}$.

Proof. Let $K_{\mathbf{x}}$ denote the union of all finite cones with the vertex $\mathbf{x} \in \partial \Omega_{F}$. Suppose Scheme (8) is stable. Choose any point $\mathbf{x} \in \Omega_{F}$. If $\mathbf{x} \in \operatorname{int}\left(\Omega_{F}\right)$, then, in view of Lemma 3, $\mathbf{F}$ in (8) will be locally Lipschitz. If $\mathbf{x} \notin \operatorname{int}\left(\Omega_{H}\right)$, then (cone property) for each $\mathbf{x}_{*} \in K_{\mathbf{x}}$ there is a straight line segment joining the points $\mathbf{x}, \mathbf{x}_{*}$. Since $\Lambda_{\Omega_{F}}\left(\mathbf{x}, \mathbf{x}_{*}\right)=\left\|\mathbf{x}_{*}-\mathbf{x}\right\|$, the necessity, in view of (14), is proven.

Conversely, suppose $\mathbf{F}$ in (8) is locally Lipschitz. Let some points $\mathbf{x}, \mathbf{x}_{*} \in \Omega_{F}$ be joined by a continuous curve $\gamma$. If $\mathbf{x}, \mathbf{x}_{*} \in \operatorname{int}\left(\Omega_{F}\right)$, then, in view of Lemma 3. we find that (14) holds. Let $\mathbf{x} \notin \operatorname{int}\left(\Omega_{F}\right)$, however $\mathbf{x}_{*} \in \operatorname{int}\left(\Omega_{F}\right)$. Choose any point $\mathbf{z} \in K_{\mathbf{x}}$ such that $\|\mathbf{x}-\mathbf{z}\|=\varepsilon$. Then, since $\mathbf{F}$ is locally Lipschitz, we have $\|\mathbf{F}(\mathbf{x})-\mathbf{F}(\mathbf{z})\| \leq C\|\mathbf{x}-\mathbf{z}\|$ for a sufficiently small $\varepsilon$. Since $\mathbf{z} \in \operatorname{int}\left(\Omega_{F}\right)$, we find, in view of Lemma 3. that $\left\|\mathbf{F}(\mathbf{z})-\mathbf{F}\left(\mathbf{x}_{*}\right)\right\| \leq C \Lambda_{\Omega_{F}}\left(\mathbf{z}, \mathbf{x}_{*}\right)$. Then

$$
\begin{gathered}
\left\|\mathbf{F}(\mathbf{x})-\mathbf{F}\left(\mathbf{x}_{*}\right)\right\| \leq\|\mathbf{F}(\mathbf{x})-\mathbf{F}(\mathbf{z})\|+\left\|\mathbf{F}(\mathbf{z})-\mathbf{F}\left(\mathbf{x}_{*}\right)\right\| \\
\leq \varepsilon C+\left\|\mathbf{F}(\mathbf{z})-\mathbf{F}\left(\mathbf{x}_{*}\right)\right\|, \quad \forall \mathbf{z}:\|\mathbf{x}-\mathbf{z}\|=\varepsilon, \forall \mathbf{s}:|\mathbf{s}| \leq s_{0} .
\end{gathered}
$$

Since $\left\|\mathbf{F}(\mathbf{x})-\mathbf{F}\left(\mathbf{x}_{*}\right)\right\|$ does not depend on $\mathbf{z}$, we find

$$
\left\|\mathbf{F}(\mathbf{x})-\mathbf{F}\left(\mathbf{x}_{*}\right)\right\| \leq \inf _{\mathbf{z}:\|\mathbf{x}-\mathbf{z}\|=\varepsilon}\left\{\varepsilon C+\left\|\mathbf{F}(\mathbf{z})-\mathbf{F}\left(\mathbf{x}_{*}\right)\right\|\right\}
$$




$$
\leq \varepsilon C+C \inf _{\mathbf{z}:\|\mathbf{x}-\mathbf{z}\|=\varepsilon} \Lambda_{\Omega_{F}}\left(\mathbf{z}, \mathbf{x}_{*}\right), \quad \forall \mathbf{s}:|\mathbf{s}| \leq s_{0} .
$$

By letting $\varepsilon \rightarrow 0$, i.e. $\mathbf{z} \rightarrow \mathbf{x}$, we find that (14) holds. The proof in the case $\mathbf{x}$, $\mathbf{x}_{*} \notin \operatorname{int}\left(\Omega_{F}\right)$ is reduced to the previous one by choosing $\mathbf{z} \in K_{\mathbf{x}_{*}}$.

We shall need the following theorem that identifies the Sobolev space $W^{1, \infty}(\Omega)$ as a space of locally Lipschitz functions.

Theorem 5 Let $\Omega_{F} \subseteq \mathbb{R}^{N}$ be open, and let $\mathbf{F}=\left\{F_{1}, F_{2}, \ldots, F_{N}\right\}^{T}$ in (8). Then, $F_{i}, i=1,2, \ldots, N$, (and, hence, $\mathbf{F}$ ) is locally Lipschitz (in the sense of having representatives) iff $F_{i} \in W^{1, \infty}\left(\Omega_{F}\right)$.

The proof of Theorem 5 can be found, e.g., in [21, Theorem 4.1], 31, p. 342]. The following lemma gives the necessary and sufficient conditions for the stability of the scheme in variations (13).

Lemma 6 Linear Scheme (13) will be stable iff there exist positive $s_{0}, C=$ const such that

$$
\left\|\mathbf{F}^{\prime}\right\| \leq C=\text { const }, \quad \forall \mathbf{x} \in \Omega_{F}, \quad \forall \mathbf{s}:|\mathbf{s}| \leq s_{0} .
$$

The proof of Lemma 6 can be found in [7] (see also, e.g., [16], 47], 48], 49]). Let us remind the well-known theorem that ensures that Lipschitz domains are extension domains (see [31, pp. 320, 356], [11, pp. 149, 285]).

Theorem 7 Let $\Omega \subset \mathbb{R}^{N}$ be an open set with uniformly Lipschitz boundary. Then there exists a continuous linear operator $\mathfrak{L}: W^{1, \infty}(\Omega) \rightarrow W^{1, \infty}\left(\mathbb{R}^{N}\right)$ such that $\mathfrak{L}(u)(\mathbf{x})=u(\mathbf{x})$ for all $u \in W^{1, \infty}(\Omega)$.

The interconnection between stability of the nonlinear scheme (8) and stability of its scheme in variations is established by the following theorem. It is assumed that $\Omega_{F}$ in (8) need not be open. It is also assumed that the set $\Omega_{F}$ is bounded, i.e. there exists $r_{0}<\infty$ such that $\Omega_{F} \subset B_{0} \equiv B\left(0, r_{0}\right)$. Let $\mathbf{F} \equiv\left\{F_{1}\right.$, $\left.F_{2}, \ldots, F_{N}\right\}^{T}$ and let $\nabla F_{i}, i=1,2, \ldots, N$, denote the distributional gradient of $F_{i}$. Definition of the distributional gradient (or distributional partial derivative) of $F_{i}$ at $\mathbf{x} \in \operatorname{int}\left(\Omega_{F}\right)$ can be found, e.g., in [31. If $\mathbf{x} \in \partial \Omega_{F}$, while $\Omega_{F}$ is Lipschitz, then the distributional gradient, $\nabla F_{i}$, is assumed to be equal to $\nabla \mathfrak{L}\left(F_{i}\right)(\mathbf{x})$.

Theorem 8 Consider the scheme (8). Let $\mathbf{F}$ be bounded and let $\Omega_{F}$ be internal path-connected, bounded, and Lipschitz. Then, the scheme, (8), will be stable in terms of Definition 2 iff its scheme in variations, (13), will be stable.

Proof. Let the scheme (8) be stable, then, in view of Theorem $4 \mathbf{F}$ will be locally Lipschitz for a common constant $C$, and, hence, in view of Theorem 5. $F_{i} \in W^{1, \infty}\left(\operatorname{int}\left(\Omega_{F}\right)\right), i=1,2, \ldots, N$. By virtue of Theorem 7 we can find $F_{i}^{*} \in W^{1, \infty}\left(\mathbb{R}^{N}\right)$ such that $F_{i}^{*}(\mathbf{x})=F_{i}(\mathbf{x})$ on $\operatorname{int}\left(\Omega_{F}\right)$. Hence $F_{i}^{*} \in W^{1, \infty}\left(B_{0}\right)$, where $B_{0}$ is an open ball such that $\Omega_{F} \subset B_{0} \subset \mathbb{R}^{N}$. Notice, $\left\|\nabla F_{i}^{*}\right\|_{\infty}, i=$ $1,2, \ldots, N$, is bounded on the ball $B_{0}$ and, naturally, on the domain $\Omega_{F} \subset B_{0}$, 
since $F_{i}^{*} \in W^{1, \infty}\left(B_{0}\right)$. In such a case there exists a common constant $C$ such that $\left\|\mathbf{F}^{\prime}\right\| \leq C$ for all $\mathbf{x} \in \Omega_{F}$, where $\left\|\mathbf{F}^{\prime}\right\| \equiv\left\|\mathbf{f}_{F}\right\|, \mathbf{f}_{F} \equiv\left\{\left\|\nabla F_{1}\right\|_{\infty},\left\|\nabla F_{2}\right\|_{\infty}\right.$, $\left.\ldots,\left\|\nabla F_{N}\right\|_{\infty}\right\}^{T}$. Hence, in view of Lemma 6, the scheme in variations, (13), is stable.

Conversely, suppose that the scheme in variations, (13), is stable. In view of Lemma 6, there exists a common constant $C$ such that $\left\|\mathbf{F}^{\prime}\right\| \leq C$ on $\Omega_{F}$, and, hence, $\left\|\nabla F_{i}\right\|_{\infty}, i=1,2, \ldots, N$, is bounded on $\Omega_{F}$. Consequently, $F_{i} \in$ $W^{1, \infty}\left(\operatorname{int}\left(\Omega_{F}\right)\right), i=1,2, \ldots, N$. Since $\operatorname{int}\left(\Omega_{F}\right)$ is an extension domain for $W^{1, \infty}\left(\right.$ int $\left.\left(\Omega_{F}\right)\right)$, we can find, in view of Theorem $7 F_{i}^{*} \in W^{1, \infty}\left(\mathbb{R}^{N}\right)$ such that $F_{i}^{*}(\mathbf{x})=F_{i}(\mathbf{x})$ on int $\left(\Omega_{F}\right)$. In view of Theorem [5, $F_{i}^{*}, i=1,2, \ldots, N$, will be locally Lipschitz on $\mathbb{R}^{N}$ (and, hence, on $\Omega_{F}$ ) for a common constant. Then $F_{i}, i=1,2, \ldots, N$, and hence $\mathbf{F}$ will be locally Lipschitz on $\Omega_{F}$, since $\Omega_{F}$ being Lipschitz satisfies the cone property. Thus, in view of Theorem 4 , the scheme (8) will be stable.

Notice, if $\mathbf{F}$ in the scheme (8) is Gateaux-differentiable, then $\nabla F_{i}$ (see Theorem 8) denotes the classical gradient of $F_{i}$, and, hence, it may be taken that $\mathbf{f}_{F}=\left\{\left\|\nabla F_{1}\right\|,\left\|\nabla F_{2}\right\|, \ldots,\left\|\nabla F_{N}\right\|\right\}^{T}$.

Remark 9 Definition 1 is "restored in its rights" for domains being internal path-connected, bounded, and Lipschitz (see the proof of Theorem 8). Namely, considering the scheme (8) with such a domain, we find that the scheme will be stable in terms of Definition Q iff it will be stable in terms of Definition 1 .

\section{Construction of stable central schemes}

In this section we consider explicit schemes on a uniform grid with time step $\Delta t$ and spatial mesh size $\Delta x$, as applied to 1-D hyperbolic equations. In view of the CFL condition [32], we assume for the explicit schemes that $\Delta t=O(\Delta x)$. Moreover, we will also assume that $\Delta x=O(\Delta t)$, since a central scheme generates, in general, a conditional approximation (see 8). In such a case, the following inequalities will be valid, for sufficiently small $\Delta t$ and $\Delta x$,

$$
\nu_{0} \Delta t \leq \Delta x \leq \mu_{0} \Delta t, \quad \nu_{0}, \mu_{0}=\text { const }, 0<\nu_{0} \leq \mu_{0} .
$$

We will focus on the following 1-D version of the problem (11):

$$
\frac{\partial \mathbf{u}}{\partial t}+\frac{\partial}{\partial x} \mathbf{f}(\mathbf{u})=0, t_{n}<t \leq t_{n+1} \equiv t_{n}+\Delta t, \quad \mathbf{u}\left(x, t_{n}\right)=\mathbf{u}^{n}(x),
$$

Since the system (22) is hyperbolic, the Jacobian matrix of $\mathbf{f}(\mathbf{u})$ possesses $M$ linearly independent eigenvectors (see, e.g., 18]). In addition, it is also assumed that

$$
\sup _{\mathbf{u} \in \Omega_{\mathbf{u}}}\|\mathbf{A}\|_{2} \leq \lambda_{\max }<\infty, \quad \mathbf{A}=\frac{\partial \mathbf{f}(\mathbf{u})}{\partial \mathbf{u}} .
$$

Using the central differencing, we write

$$
\left.\frac{\partial \mathbf{u}}{\partial t}\right|_{t=t_{n+0.25}, x=x_{i+0.5}}=\frac{\mathbf{u}_{i+0.5}^{n+0.5}-\mathbf{u}_{i+0.5}^{n}}{0.5 \Delta t}+O\left((\Delta t)^{2}\right),
$$




$$
\left.\frac{\partial \mathbf{f}}{\partial x}\right|_{t=t_{n+0.25}, x=x_{i+0.5}}=\frac{\mathbf{f}_{i+1}^{n+0.25}-\mathbf{f}_{i}^{n+0.25}}{\Delta x}+O\left((\Delta x)^{2}\right) .
$$

By virtue of (24)-(25) we approximate (22) on the cell $\left[x_{i}, x_{i+1}\right] \times\left[t_{n}, t_{n+0.5}\right]$ by the following difference equation

$$
\mathbf{v}_{i+0.5}^{n+0.5}=\mathbf{v}_{i+0.5}^{n}-\frac{\Delta t}{2 \Delta x}\left(\mathbf{g}_{i+1}^{n+0.25}-\mathbf{g}_{i}^{n+0.25}\right) .
$$

As usual, the mathematical treatment for the second step (i.e., on the cell $\left.\left[x_{i-0.5}, x_{i+0.5}\right] \times\left[t_{n+0.5}, t_{n+1}\right]\right)$ of a staggered scheme will, in general, not be included in the text, because it is quite similar to the treatment for the first step.

Considering that (26) approximates (22) with the accuracy $O\left((\Delta x)^{2}+(\Delta t)^{2}\right)$, the next problem is to approximate $\mathbf{v}_{i+0.5}^{n}$ and $\mathbf{g}_{i}^{n+0.25}$ in such a way as to retain the accuracy of the approximation. For instance, the following approximations

$$
\mathbf{v}_{i+0.5}^{n}=0.5\left(\mathbf{v}_{i}^{n}+\mathbf{v}_{i+1}^{n}\right)+O\left((\Delta x)^{2}\right), \quad \mathbf{g}_{i}^{n+0.25}=\mathbf{f}\left(\mathbf{v}_{i}^{n}\right)+O(\Delta t),
$$

leads to the staggered form of the famed LxF scheme that is of the first-order approximation (see, e.g., [18, p. 170]). One way to obtain a higher-order scheme is to use a higher order interpolation. At the same time it is required of the interpolant to be monotonicity preserving. Notice, the classic cubic spline [46] does not possess such a property (see Figure 1 $\mathrm{a}$ ). In the following, subsection 3.1. we consider the problem of high-order interpolation of $\mathbf{v}_{i+0.5}^{n}$ in (26) with closer inspection.

\subsection{Monotone $C^{1}$ piecewise cubics in construction of ex- plicit schemes}

We will consider some theoretical aspects for high-order interpolation and employment of monotone $C^{1}$ piecewise cubics (see, e.g., [14, 27]) in construction of monotone explicit schemes.

Let $\mathbf{p}=\mathbf{p}(x) \equiv\left\{p^{1}(x), \ldots, p^{k}(x), \ldots, p^{m}(x)\right\}^{T}$ be an interpolant, and let

$$
\begin{gathered}
\mathbf{p}_{i}=\mathbf{p}\left(x_{i}\right), \quad \mathbf{p}_{i}^{\prime}=\mathbf{p}^{\prime}\left(x_{i}\right), \quad \Delta \mathbf{p}_{i}=\mathbf{p}_{i+1}-\mathbf{p}_{i}, \\
\mathbf{p}_{i}^{\prime}=\mathbb{A}_{i} \cdot \frac{\Delta \mathbf{p}_{i}}{\Delta x}, \quad \mathbf{p}_{i+1}^{\prime}=\mathbb{B}_{i} \cdot \frac{\Delta \mathbf{p}_{i}}{\Delta x}
\end{gathered}
$$

where $\mathbf{p}_{i}^{\prime}$ denotes the derivative of the interpolant at $x=x_{i}$. The diagonal matrices $\mathbb{A}_{i}$ and $\mathbb{B}_{i}$ in (28) are defined as follows

$$
\mathbb{A}_{i}=\operatorname{diag}\left\{\alpha_{i}^{1}, \alpha_{i}^{2}, \ldots, \alpha_{i}^{m}\right\}, \mathbb{B}_{i}=\operatorname{diag}\left\{\beta_{i}^{1}, \beta_{i}^{2}, \ldots, \beta_{i}^{m}\right\} .
$$

If $\mathbf{p}=\mathbf{p}(x)$ is a $C^{1}$ piecewise cubic interpolant (see, e.g., [14], [27]), then it will be component-wise monotone on $\left[x_{i}, x_{i+1}\right]$ iff one of the following conditions (see [14, 27]) is satisfied:

$$
\left(\alpha_{i}^{k}-1\right)^{2}+\left(\alpha_{i}^{k}-1\right)\left(\beta_{i}^{k}-1\right)+\left(\beta_{i}^{k}-1\right)^{2}-3\left(\alpha_{i}^{k}+\beta_{i}^{k}-2\right) \leq 0,
$$




$$
\alpha_{i}^{k}+\beta_{i}^{k} \leq 3, \quad \alpha_{i}^{k} \geq 0, \beta_{i}^{k} \geq 0, \quad \forall i, k .
$$

The region of monotonicity is shown in Figure $1 \mathrm{~b}$. The results of implementing a monotone $C^{1}$ piecewise cubic interpolation when compared with the classic cubic spline interpolation, are depicted in Figure 1a.
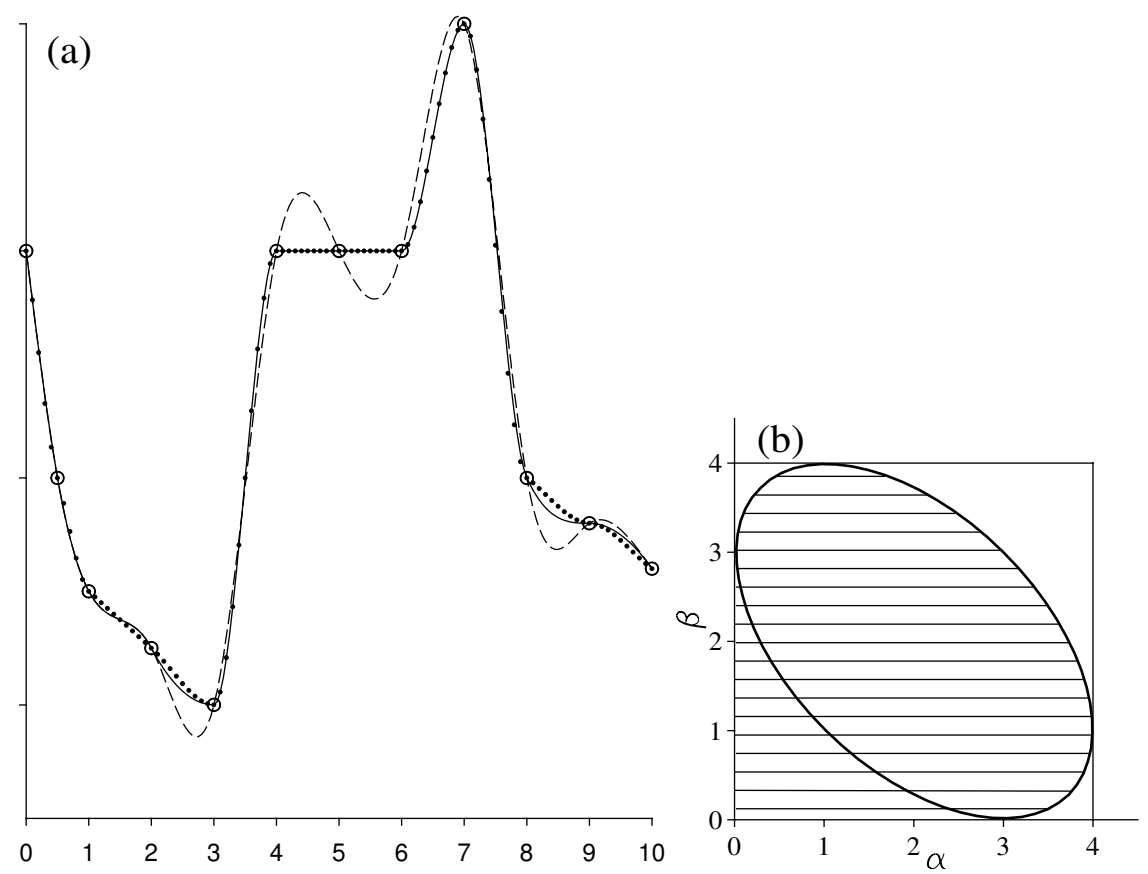

Figure 1: Monotone piecewise cubic interpolation. (a) Interpolation of a 1-D tabulated function. Circles: prescribed tabulated values; Dashed line: classic cubic spline; Solid line: monotone piecewise cubic under $\alpha, \beta \leq 3$; Dotted line: monotone piecewise cubic under $\alpha, \beta \leq 1$. (b) Necessary and sufficient conditions for monotonicity. Horizontal hatching: region of monotonicity; Unshaded: cubic is non-monotone.

We note (Figure 1 $1 \mathrm{a}$ ) that the constructed function produces monotone interpolation and has a small, or even practically zero, deviation from the classic cubic spline at some sections where the classic cubic spline is monotone.

Let us consider the problem of monotone high-order approximation of $\mathbf{v}_{i+0.5}^{n}$ in (26). First, given the values, $\mathbf{p}_{i}$ and $\mathbf{p}_{i+1}$, of the interpolant $\mathbf{p}=\mathbf{p}(x)$ and the approximate estimates, $\mathbf{d}_{i}$ and $\mathbf{d}_{i+1}$, of its derivatives, namely

$$
\mathbf{p}_{i}^{\prime}=\mathbf{d}_{i}+O\left((\Delta x)^{s}\right),
$$


we obtain the following interpolation formula

$$
\mathbf{p}_{i+0.5}=0.5\left(\mathbf{p}_{i}+\mathbf{p}_{i+1}\right)-\frac{\Delta x}{8}\left(\mathbf{d}_{i+1}-\mathbf{d}_{i}\right)+O\left((\Delta x)^{r}\right)
$$

where

$$
r=\min (4, s+1) \text {. }
$$

Actually, let $\mathbf{p}(x)$ be sufficiently smooth, then, using the Taylor series expansion of the function $\mathbf{p}(x)$, we obtain:

$$
\mathbf{p}_{i+1}+\mathbf{p}_{i}=2 \mathbf{p}_{i+0.5}+\mathbf{p}_{i+05}^{\prime \prime}\left(\frac{\Delta x}{2}\right)^{2}+\frac{\mathbf{p}_{i+05}^{\prime \prime \prime \prime}}{12}\left(\frac{\Delta x}{2}\right)^{4}+O\left((\Delta x)^{6}\right)
$$

In a similar manner, using the Taylor series expansion of the function $\mathbf{p}^{\prime}(x)$, we can show that

$$
\mathbf{p}_{i+1}^{\prime}-\mathbf{p}_{i}^{\prime}=\mathbf{p}_{i+05}^{\prime \prime} \Delta x+\frac{\mathbf{p}_{i+05}^{\prime \prime \prime \prime}}{3}\left(\frac{\Delta x}{2}\right)^{3}+O\left((\Delta x)^{5}\right)
$$

By virtue of (36) we obtain from (35) that

$$
\mathbf{p}_{i+0.5}=0.5\left(\mathbf{p}_{i}+\mathbf{p}_{i+1}\right)-\frac{\Delta x}{8}\left(\mathbf{p}_{i+1}^{\prime}-\mathbf{p}_{i}^{\prime}\right)+\frac{\mathbf{p}_{i+05}^{\prime \prime \prime \prime}}{24}\left(\frac{\Delta x}{2}\right)^{4}+O\left((\Delta x)^{6}\right)
$$

In view of (32) we obtain from (37) the following interpolation formula

$$
\mathbf{p}_{i+0.5}=0.5\left(\mathbf{p}_{i}+\mathbf{p}_{i+1}\right)-\frac{\Delta x}{8}\left(\mathbf{d}_{i+1}-\mathbf{d}_{i}\right)+O\left((\Delta x)^{4}+(\Delta x)^{s+1}\right) .
$$

Hence, by virtue of (38) and (33), we conclude that (34) is, in general, valid. It is clear that if (32) is valid and $\mathbf{d}_{i+1}-\mathbf{d}_{i}=\left(\mathbf{p}_{i+1}^{\prime}-\mathbf{p}_{i}^{\prime}\right)+O\left((\Delta x)^{s+1}\right)$, then $r=\min (4, s+2)$ in (33). Thus, if $\mathbf{p}(x)$ has a continuous fourth derivative and $\mathbf{p}_{i}^{\prime}$ can be estimated with accuracy $O\left((\Delta x)^{3}\right)$ (see, e.g., [26, p. 112]), then $r=4$ in (33).

Let us note that instead of point values employed in the construction of the scheme (26), it can be used the cell averages (see, e.g., [5], [29], 32]). Then, given the values, $\overline{\mathbf{p}}_{i}$, of the averaged interpolant $\overline{\mathbf{p}}=\overline{\mathbf{p}}(x)$ and the estimates, $\overline{\mathbf{d}}_{i}$ and $\overline{\mathbf{d}}_{i+1}$, of its derivatives, namely $\overline{\mathbf{p}}_{i}^{\prime}=\overline{\mathbf{d}}_{i}+O\left((\Delta x)^{s}\right)$ we obtain the same formula (33) written for the averaged values. Further, to estimate the flux at $x_{i}$, the point value $\mathbf{p}_{i}$ can be reconstructed using a piecewise polynomial interpolation (e.g., [5, 29]). Notice, we need not any reconstruction when dealing with first- and second-order schemes, since the point values agree with the corresponding cell averages to $O\left((\Delta x)^{2}\right)$ (e.g., [29], [32]). We will therefore omit the bar notation when dealing with such schemes.

Instead of (33), we will employ the following interpolation formula

$$
\mathbf{p}_{i+0.5}=0.5\left(\mathbf{p}_{i}+\mathbf{p}_{i+1}\right)-\varkappa \frac{\Delta x}{8}\left(\mathbf{d}_{i+1}-\mathbf{d}_{i}\right), \quad 0 \leq \varkappa \leq 1
$$


where, for some reason, a new parameter $\varkappa$ is used. Consider, for instance, the case when the point value $\mathbf{v}_{i+0.5}^{n}$ in the scheme (26) is estimated by the cell average calculated on the basis of the monotone $C^{1}$ piecewise cubics. Note that this estimation is of second-order. Such an approach leads to the interpolation of the point value by formula (39) with the parameter $\varkappa=2 / 3$. Obviously, the interpolation (39) is of second-order under $0 \leq \varkappa<1$, and (39) coincides with (33) under $\varkappa=1$ to give fourth order accuracy. Notice, if $0<\varkappa<1$, then $\mathbf{p}_{i+0.5}$ can be estimated with accuracy $O\left((\Delta x)^{4}\right)$ by (39) provided that $1-\varkappa=O\left((\Delta x)^{2}\right)$. Actually, by virtue of (36) and (32), we obtain that

$$
\begin{gathered}
\varkappa \frac{\Delta x}{8}\left(\mathbf{d}_{i+1}-\mathbf{d}_{i}\right)=\frac{\Delta x}{8}\left(\mathbf{d}_{i+1}-\mathbf{d}_{i}\right)+(1-\varkappa) \frac{(\Delta x)^{2}}{8} \frac{\mathbf{d}_{i+1}-\mathbf{d}_{i}}{\Delta x}=\frac{\Delta x}{8}\left(\mathbf{d}_{i+1}-\mathbf{d}_{i}\right) \\
+\frac{O\left((\Delta x)^{4}\right)}{8} \mathbf{p}_{i+05}^{\prime \prime}+O\left((\Delta x)^{s+3}\right)=\frac{\Delta x}{8}\left(\mathbf{d}_{i+1}-\mathbf{d}_{i}\right)+O\left((\Delta x)^{4}\right)
\end{gathered}
$$

The approximation of derivatives $\mathbf{p}_{i}^{\prime}$ can be done by the following three steps 14]: (i) initialization of the derivatives $\mathbf{p}_{i}^{\prime}=\mathbf{d}_{i}+O\left((\Delta x)^{s}\right)$; (ii) choice of subregion of monotonicity; (iii) modification of the initialized derivatives, $\mathbf{d}_{i}$, to produce a monotone interpolant.

The matter of initialization of the derivatives is the most subtle issue of this algorithm. Thus, using the two-point or the three-point (centered) difference formula (e.g. 27, 41]) we obtain, in general, second-order approximation. Performing the initialization of the derivatives $\mathbf{p}_{i}^{\prime}=\mathbf{d}_{i}+O\left((\Delta x)^{s}\right)$ in the interpolation formula (39) by the classic cubic spline [46] interpolation, we obtain $s=3$ (e.g., [26, [27]). The same accuracy can be achieved by using the fourpoint approximation [27. However, the efficiency of the algorithm based on the classic cubic spline interpolation is comparable with the one based on the four-point approximation, as the number of multiplications and divisions per one node is approximately the same for both algorithms.

Obviously, for each interval $\left[x_{i}, x_{i+1}\right]$ in which the initialized derivatives $\mathbf{d}_{i}$, $\mathbf{d}_{i+1}$ such that at least one point $\left(\alpha_{i}^{k}, \beta_{i}^{k}\right)$ does not belong to the region of monotonicity (30)-(31), the derivatives $\mathbf{d}_{i}, \mathbf{d}_{i+1}$ must be modified to $\widetilde{\mathbf{d}}_{i}, \widetilde{\mathbf{d}}_{i+1}$ such that the point $\left(\widetilde{\alpha}_{i}^{k}, \widetilde{\beta}_{i}^{k}\right)$ will be in the region of monotonicity. Moreover, it is desirable to modify the derivatives $\mathbf{d} \equiv\left\{\ldots, \mathbf{d}_{i-1}^{T}, \mathbf{d}_{i}^{T}, \mathbf{d}_{i+1}^{T}, \ldots\right\}^{T}$ in such a way that $\widetilde{\mathbf{d}} \equiv\left\{\ldots, \widetilde{\mathbf{d}}_{i-1}^{T}, \widetilde{\mathbf{d}}_{i}^{T}, \widetilde{\mathbf{d}}_{i+1}^{T}, \ldots\right\}^{T}$ would be the solution to the following mathematical programming problem:

$$
\|\mathbf{d}-\widetilde{\mathbf{d}}\| \rightarrow \min _{\widetilde{\mathbf{d}}}
$$

The modification of the initialized derivatives, would be much simplified if we take a square as a subregion of monotonicity. In connection with this, we will make use the subregions of monotonicity represented in the following form:

$$
0 \leq \alpha_{i}^{k} \leq 4 \aleph, \quad 0 \leq \beta_{i}^{k} \leq 4 \aleph, \quad \forall i, k,
$$


where $\aleph$ is a monotonicity parameter. If $\aleph=0.75$, then the subregion (42), called de Boor-Swartz box [27, coincides with the one used by Fritsch and Carlson [14. It is proven in [7] that the interpolation (39) will be monotone, i.e. the value of an arbitrary component of $\mathbf{p}_{i+0.5}$ will be between the corresponding components of $\mathbf{p}_{i}$ and $\mathbf{p}_{i+1}$, iff (42) will be valid provided that $0 \leq \aleph \leq 1$. Notice, in such a case the point $\left(\alpha_{i}^{k}, \beta_{i}^{k}\right)$ may be out of the region of monotonicity shown in Figure $1 \mathrm{~b}$.

To fulfill the conditions of monotonicity (42), the modification of derivatives $\mathbf{d}_{i}=\left\{d_{i}^{1}, d_{i}^{2}, \ldots, d_{i}^{m}\right\}$ can be done by the following algorithm suggested, in fact, by Fritsch and Carlson [14] (see also [27]):

$$
S_{i}^{k}:=4 \aleph \min \bmod \left(\Delta_{i-1}^{k}, \Delta_{i}^{k}\right), \quad \widetilde{d}_{i}^{k}:=\min \bmod \left(d_{i}^{k}, S_{i}^{k}\right), \quad \aleph=\text { const },
$$

where $\Delta_{i}^{k}=\left(p_{i+1}^{k}-p_{i}^{k}\right) / \Delta x$, the function $\min \bmod (x, y)$ is defined (e.g., [27], [29], 36], [41], [50]) as follows

$$
\min \bmod (x, y) \equiv \frac{1}{2}[\operatorname{sgn}(x)+\operatorname{sgn}(y)] \min (|x|,|y|) .
$$

\subsection{Construction of nonstaggered central schemes}

We will consider explicit central schemes on a uniform grid with time step $\Delta t$ and spatial mesh size $\Delta x$. In view of the interpolation formula (39), the staggered scheme (26) becomes

$$
\mathbf{v}_{i+0.5}^{n+0.5}=0.5\left(\mathbf{v}_{i+1}^{n}+\mathbf{v}_{i}^{n}\right)-\varkappa \frac{\Delta x}{8}\left(\mathbf{d}_{i+1}^{n}-\mathbf{d}_{i}^{n}\right)-\frac{\Delta t}{2} \frac{\mathbf{f}\left(\mathbf{v}_{i+1}^{n}\right)-\mathbf{f}\left(\mathbf{v}_{i}^{n}\right)}{\Delta x}
$$

where $\mathbf{d}_{i}^{n}$ denotes the derivative of the interpolant at $x=x_{i}$, the range of values for the parameter $\varkappa$ is the segment $0 \leq \varkappa \leq 1$. If $\varkappa=0$, then the scheme (45) coincides with the LxF scheme. It is shown in [7] that the first-order, $O\left(\Delta t+(\Delta x)^{r} / \Delta t+(\Delta x)^{2}\right)$, scheme (45) generates a conditional approximation, because it approximates (22) only if $(\Delta x)^{r} / \Delta t \rightarrow 0$ as $\Delta x \rightarrow 0$ and $\Delta t \rightarrow 0$, where $r$ is the order of approximation of $\mathbf{v}_{i+0.5}^{n}$ by the interpolation formula (39). The scheme (45) is abbreviated in [8] as COS1.

Let us develop new nonoscillatory central schemes, which are based on regular, nonstaggered spatial grids. Using the central differencing, we approximate (22) on the cell $\left[x_{i-1}, x_{i+1}\right] \times\left[t_{n}, t_{n+1}\right]$ by the following difference equation

$$
\mathbf{v}_{i}^{n+1}=\mathbf{v}_{i}^{n}-\frac{\Delta t}{2 \Delta x}\left(\mathbf{g}_{i+1}^{n+0.5}-\mathbf{g}_{i-1}^{n+0.5}\right) .
$$

Notice, the approximation $\mathbf{g}_{i}^{n+0.5}=\mathbf{f}\left(\mathbf{v}_{i}^{n}\right)+O(\Delta t)$, leads to the first-order, $O\left(\Delta t+(\Delta x)^{2}\right)$, scheme

$$
\mathbf{v}_{i}^{n+1}=\mathbf{v}_{i}^{n}-\frac{\Delta t}{2 \Delta x}\left(\mathbf{f}_{i+1}^{n}-\mathbf{f}_{i-1}^{n}\right)
$$


which is absolutely unstable [16, p. 113], [32, p. 71]. Let us construct a stable scheme on the basis of (46). The interpolation formula (39), as applied to the cell $\left[x_{i-1}, x_{i+1}\right] \times\left[t_{n}, t_{n+1}\right]$, becomes

$$
\mathbf{p}_{i}=0.5\left(\mathbf{p}_{i-1}+\mathbf{p}_{i+1}\right)-\varkappa \frac{\Delta x}{4}\left(\mathbf{d}_{i+1}-\mathbf{d}_{i-1}\right) .
$$

Equality (48), after elementary transformations, leads to

$$
\mathbf{p}_{i}=0.25\left(\mathbf{p}_{i-1}+2 \mathbf{p}_{i}+\mathbf{p}_{i+1}\right)-\varkappa \frac{\Delta x}{8}\left(\mathbf{d}_{i+1}-\mathbf{d}_{i-1}\right) .
$$

Using (49) we obtain from (47) the following nonstaggered central scheme

$$
\mathbf{v}_{i}^{n+1}=\frac{\mathbf{v}_{i+1}^{n}+2 \mathbf{v}_{i}^{n}+\mathbf{v}_{i-1}^{n}}{4}-\varkappa \frac{\Delta x}{8}\left(\mathbf{d}_{i+1}^{n}-\mathbf{d}_{i-1}^{n}\right)-\frac{\Delta t}{2 \Delta x}\left(\mathbf{f}_{i+1}^{n}-\mathbf{f}_{i-1}^{n}\right) .
$$

where $0 \leq \varkappa \leq 1$. In view of (32)-(34), as applied to the cell $\left[x_{i-1}, x_{i+1}\right] \times$ $\left[t_{n}, t_{n+1}\right]$, the local truncation error, $\psi$, on a sufficiently smooth solution $\mathbf{u}(x, t)$ to (22) is found to be

$$
\psi=O\left(\Delta t+(\Delta x)^{2}\right)+O\left(\frac{(\Delta x)^{r}}{\Delta t}\right)+(1-\varkappa) O\left(\frac{(\Delta x)^{2}}{\Delta t}\right),
$$

where $r=\min (4, s+1), s$ denotes the order of approximation in (32). Note that the scheme (50) will be $O\left(\Delta t+(\Delta x)^{2}\right)$ accurate if $r \geq 3$ as well as $1-\varkappa=$ $O(\Delta x)$. The first-order nonstaggered central scheme (50), being a monotone approximation of the 1-D equation (22) by $C^{1}$ cubics, will be abbreviated to MAC1.

Let us consider the transformation from the scheme (47) to (50) with closer inspection. We will use the, so called, first differential approximation of the scheme (47) (15, p. 45], 49, p. 376]; see also 'modified equations' in [15, p. 45], 32, 35). As reported in [15, 49, this heuristic method was originally presented by Hirt (1968) (see [15, p. 45]) as well as by Shokin and Yanenko (1968) (see [49, p. 376]), and has since been widely employed in the development of stable difference schemes for PDEs.

The first differential approximation of the scheme (47) is the following:

$$
\frac{\partial \mathbf{u}}{\partial t}+\frac{\partial}{\partial x} \mathbf{f}(\mathbf{u})=-\frac{\Delta t}{2} \frac{\partial}{\partial x}\left(\mathbf{A}^{2} \cdot \frac{\partial \mathbf{u}}{\partial x}\right), \quad \mathbf{A}^{2} \equiv \frac{\partial \mathbf{f}}{\partial \mathbf{u}} \cdot \frac{\partial \mathbf{f}}{\partial \mathbf{u}} .
$$

The negative diffusion term in (52) is associated with instability, as this therm is the source of energy resulting in an unlimited growth of the amplitude of the solution (see, e.g., [51, 52]). Notice, for the sake of convenience we use the same notation in (22) and in (52) in spite of the fact that these equations are different. We will use such an approach if it does not lead to confusion.

To obtain (50), we, in fact, added to the right-hand side of (47) the value

$$
\mathbf{E}_{i} \equiv \frac{\mathbf{v}_{i+1}^{n}-2 \mathbf{v}_{i}^{n}+\mathbf{v}_{i-1}^{n}}{4}-\varkappa \frac{\Delta x}{8}\left(\mathbf{d}_{i+1}^{n}-\mathbf{d}_{i-1}^{n}\right) .
$$


Let the interpolant $\mathbf{p}=\mathbf{p}(x)$ be such that $\mathbf{p}_{i}=\mathbf{v}_{i}^{n}$. If $\varkappa=1$ and $\mathbf{d}_{i}=\mathbf{p}_{i}^{\prime}$, then, by virtue of (32)-(37) as applied to the cell $\left[x_{i-1}, x_{i+1}\right] \times\left[t_{n}, t_{n+1}\right]$, we find that

$$
\mathbf{E}_{i}=-\frac{(\Delta x)^{4}}{48} \mathbf{p}_{i}^{\prime \prime \prime \prime}+O\left((\Delta x)^{6}\right) .
$$

Thus, when we add $\mathbf{E}_{i}$ of (53) to the right-hand side of (47), we, in fact, add the negative fourth-order diffusion term of (54) to the right-hand side of the first differential approximation (52). Such a term, in contrast to a negative secondorder diffusion term, stabilizes the system and produces dissipative effect (see, e.g., [51, 52]).

If $\varkappa=1$ and $\mathbf{p}_{i}^{\prime}$ is estimated by the central-difference derivative, i.e. $\mathbf{d}_{i}=$ $\left(\mathbf{p}_{i+1}-\mathbf{p}_{i-1}\right) /(2 \Delta x)$, then

$$
\mathbf{p}_{i}^{\prime}=\mathbf{d}_{i}-\frac{(\Delta x)^{2}}{6} \mathbf{p}_{i}^{\prime \prime \prime}+O\left((\Delta x)^{4}\right) .
$$

In such a case we obtain, instead of (54), that

$$
\mathbf{E}_{i}=-\frac{3(\Delta x)^{4}}{48} \mathbf{p}_{i}^{\prime \prime \prime \prime}+O\left((\Delta x)^{5}\right) .
$$

We can see from (56) that the estimation of the derivative $\mathbf{p}_{i}^{\prime}$ by the centraldifference derivative leads to the same order of accuracy as it was in the previous example when $\mathbf{d}_{i}=\mathbf{p}_{i}^{\prime}$. Furthermore, the scheme (50) will be more dissipative in such a case, since the coefficient of the fourth-order diffusion term in (56) is three times bigger than the similar coefficient in (54).

If $0 \leq \varkappa \leq 1$ and $\mathbf{p}_{i}^{\prime}$ is estimated by the central-difference derivative, then we find

$$
\mathbf{E}_{i}=(1-\varkappa) \frac{(\Delta x)^{2}}{4} \mathbf{p}_{i}^{\prime \prime}+(1-4 \varkappa) \frac{(\Delta x)^{4}}{48} \mathbf{p}_{i}^{\prime \prime \prime \prime}+O\left((\Delta x)^{5}\right) .
$$

Note that the estimations (54), (56), and (57) will be valid if the derivative $\mathbf{d}_{i}$ need not be modified, i.e. (42) will be valid without implementing the algorithm (43). Otherwise, there is a risk to add negative second-order diffusion terms to the right-hand side of (52). Let us consider, for instance, the case when $\varkappa=1$ and the derivative $\mathbf{p}_{i+1}^{\prime}$ is estimated by the right-difference derivative, i.e. $\mathbf{d}_{i+1}=\left(\mathbf{p}_{i+2}-\mathbf{p}_{i+1}\right) / \Delta x$, however $\mathbf{p}_{i-1}^{\prime}$ is estimated by the central-difference derivative. In such a case we obtain:

$$
\mathbf{E}_{i}=-\frac{(\Delta x)^{2}}{16} \mathbf{p}_{i+1}^{\prime \prime}-\frac{1.25(\Delta x)^{4}}{16} \mathbf{p}_{i+1}^{\prime \prime \prime \prime}+O\left((\Delta x)^{5}\right) .
$$

In view of (58) and (57), we conclude that the part played by the parameter $\varkappa$ in suppressing spurious oscillations produced by negative second-order diffusion terms can be very important.

Interestingly, there is a possibility to improve the scheme (45) by introducing an additional positive numerical viscosity, i.e., using the vanishing viscosity 
method [18, [32, such that the scheme's order of accuracy would increase up to $O\left((\Delta t)^{2}+(\Delta x)^{2}\right)$. Such an approach [8] leads to the following second order central scheme

$$
\begin{aligned}
\mathbf{v}_{i+0.5}^{n+0.5}= & 0.5\left(\mathbf{v}_{i+1}^{n}+\mathbf{v}_{i}^{n}\right)-\varkappa \frac{\Delta x}{8}\left(\mathbf{d}_{i+1}^{n}-\mathbf{d}_{i}^{n}\right)-\frac{\Delta t}{2} \frac{\mathbf{f}\left(\mathbf{v}_{i+1}^{n}\right)-\mathbf{f}\left(\mathbf{v}_{i}^{n}\right)}{\Delta x} \\
& +\frac{(\Delta t)^{2}}{8 \Delta x}\left[\left(\mathbf{A}_{i+1}^{n}\right)^{2} \cdot \mathbf{d}_{i+1}^{n}-\left(\mathbf{A}_{i}^{n}\right)^{2} \cdot \mathbf{d}_{i}^{n}\right], \quad \mathbf{A} \equiv \frac{\partial \mathbf{f}}{\partial \mathbf{u}},
\end{aligned}
$$

where $\mathbf{d}_{i}^{n}$ is the derivative of the interpolant at $x=x_{i}$. The scheme (59) is abbreviated in [8] as COS2.

Let us develop a nonstaggered central scheme that will be of second order. Using the central differencing, we approximate (22) at the point $x=x_{i}, t=$ $t_{n+05}$ by the following equation:

$$
\mathbf{v}_{i}^{n+1}=\mathbf{v}_{i}^{n}-\left.\Delta t \frac{\partial \mathbf{f}}{\partial x}\right|_{x=x_{i}, t=t_{n+05}} .
$$

Using Taylor series expansion, we approximate $\mathbf{g}_{i}^{n+0.5}$ in (46) with the accuracy $O\left((\Delta t)^{2}\right)$ :

$$
\mathbf{g}_{i}^{n+0.5}=\mathbf{f}\left(\mathbf{v}_{i}^{n}\right)+\left.\frac{\partial \mathbf{f}\left(\mathbf{v}_{i}^{n}\right)}{\partial t}\right|_{t=t_{n}} \frac{\Delta t}{2}+O\left(\Delta t^{2}\right) .
$$

By virtue of the PDE system, (22), we find

$$
\frac{\partial \mathbf{f}}{\partial t}=\frac{\partial \mathbf{f}}{\partial \mathbf{u}} \cdot \frac{\partial \mathbf{u}}{\partial t}=-\frac{\partial \mathbf{f}}{\partial \mathbf{u}} \cdot \frac{\partial \mathbf{f}}{\partial \mathbf{u}} \cdot \frac{\partial \mathbf{u}}{\partial x}=-\left(\frac{\partial \mathbf{f}}{\partial \mathbf{u}}\right)^{2} \cdot \frac{\partial \mathbf{u}}{\partial x} .
$$

By virtue of (49) and (61)-(62), we find

$$
\begin{aligned}
\mathbf{v}_{i}^{n+1}= & \frac{\mathbf{v}_{i-1}^{n}+2 \mathbf{v}_{i}^{n}+\mathbf{v}_{i+1}^{n}}{4}-\varkappa \frac{\Delta x}{8}\left(\mathbf{d}_{i+1}^{n}-\mathbf{d}_{i-1}^{n}\right)-\left.\Delta t \frac{\partial \mathbf{f}}{\partial x}\right|_{x=x_{i}, t=t_{n}} \\
& +\left.\frac{(\Delta t)^{2}}{2} \frac{\partial}{\partial x}\left(\mathbf{A}^{2} \frac{\partial \mathbf{u}}{\partial x}\right)\right|_{x=x_{i}, t=t_{n}}, \quad \mathbf{A}^{2} \equiv \frac{\partial \mathbf{f}}{\partial \mathbf{u}} \cdot \frac{\partial \mathbf{f}}{\partial \mathbf{u}} .
\end{aligned}
$$

Then, approximating the last two terms in the right-hand side of (63) with the second order accuracy, we obtain

$$
\begin{gathered}
\mathbf{v}_{i}^{n+1}=\frac{\mathbf{v}_{i-1}^{n}+2 \mathbf{v}_{i}^{n}+\mathbf{v}_{i+1}^{n}}{4}-\varkappa \frac{\Delta x}{8}\left(\mathbf{d}_{i+1}^{n}-\mathbf{d}_{i-1}^{n}\right)-\frac{\Delta t}{2 \Delta x}\left(\mathbf{f}_{i+1}^{n}-\mathbf{f}_{i-1}^{n}\right) \\
+\frac{\varsigma(\Delta t)^{2}}{2(\Delta x)^{2}}\left[\mathbf{D}_{i+0.5}^{n} \cdot\left(\mathbf{v}_{i+1}^{n}-\mathbf{v}_{i}^{n}\right)-\mathbf{D}_{i-0.5}^{n} \cdot\left(\mathbf{v}_{i}^{n}-\mathbf{v}_{i-1}^{n}\right)\right], \varsigma=\text { const } \geq 0,
\end{gathered}
$$

where $\mathbf{D}_{i+0.5}^{n}=0.5\left[\left(\mathbf{A}_{i}^{n}\right)^{2}+\left(\mathbf{A}_{i+1}^{n}\right)^{2}\right]$, the parameter $\varsigma$ is introduced by analogy with $\varkappa$ in (45). Scheme (64) coincides with the scheme MAC1, (50), provided that $\varsigma=0$. The last term in right-hand side of (64) can be seen as the 
non-negative numerical viscosity introduced into the first order scheme (50). Thus, we are dealing with the vanishing viscosity method [18], 32] and, hence, in view of [18, Theorem 3.3], the scheme, (64), satisfies the entropy condition. To make this point a little bit more clear, we consider a viscous perturbation of the system (22). We associate with (22) the following parabolic system

$$
\frac{\partial \mathbf{u}_{\epsilon}}{\partial t}+\frac{\partial}{\partial x} \mathbf{f}\left(\mathbf{u}_{\epsilon}\right)=\epsilon \frac{\partial}{\partial x}\left(\mathbf{A}^{2} \cdot \frac{\partial \mathbf{u}_{\epsilon}}{\partial x}\right), \quad \epsilon>0
$$

where the right-hand side can be viewed as a viscosity term. It is assumed that Mock's assumption of admissibility [18, p. 32] is valid, i.e. the matrix $U^{\prime \prime} \cdot \mathbf{A}^{2}$ is positive-definite. Here $U=U\left(\mathbf{u}_{\epsilon}\right)$ denotes a strictly convex entropy, $U^{\prime \prime}$ denotes the Hessian matrix of $U$. We recover solutions of (22) as the limits of solutions to (65) as $\epsilon \rightarrow 0$. If we divide the scheme COS1, (50), by $\Delta t$ and group all the terms of this scheme together in its left-hand side, then this group, in view of the above, is the finite difference approximation of the left-hand side of Eq. (65) on the cell $\left[x_{i-1}, x_{i+1}\right] \times\left[t_{n}, t_{n+1}\right]$. The right-hand side of Eq. (65) is approximated as following

$$
\epsilon \frac{\partial}{\partial x}\left(\mathbf{A}^{2} \cdot \frac{\partial \mathbf{u}_{\epsilon}}{\partial x}\right) \approx \epsilon \frac{\mathbf{D}_{i+0.5}^{n} \cdot\left(\mathbf{v}_{i+1}^{n}-\mathbf{v}_{i}^{n}\right)-\mathbf{D}_{i-0.5}^{n} \cdot\left(\mathbf{v}_{i}^{n}-\mathbf{v}_{i-1}^{n}\right)}{(\Delta x)^{2}}
$$

where $\mathbf{D}_{i \pm 0.5}^{n}=0.5\left[\left(\mathbf{A}_{i}^{n}\right)^{2}+\left(\mathbf{A}_{i \pm 1}^{n}\right)^{2}\right]$. If $\epsilon=\varsigma \Delta t / 2$, then we obtain the scheme (64) as the approximation of Eq. (65) on the cell $\left[x_{i-1}, x_{i+1}\right] \times\left[t_{n}, t_{n+1}\right]$. Thus, owing to the last term in the right-hand side of (64), the grid function $\mathbf{v}_{i}^{n}$ in (64) can be considered as Lipschitz-continuous. Moreover, owing to this term, the scheme (64) is $O\left((\Delta x)^{2}+(\Delta t)^{2}\right)$ accurate provided that $\varkappa=\varsigma=1$. The nonstaggered central scheme (64), being a monotone approximation of the 1-D equation (22) with the second order, will be abbreviated to MAC2.

\subsection{Stability of the developed schemes}

It is proven in [8] that the scheme COS2, (59), will be, in general, stable if

$$
\left|\varkappa-\varsigma C_{r}^{2}\right| \aleph+C_{r} \leq 1, \quad C_{r}=\frac{\Delta t \lambda_{\max }}{\Delta x} .
$$

Let us note that the stability condition (67) is not exactly correct, namely, there exist infrequent situations when (67) is valid nevertheless the scheme is not stable provided $\varkappa, \varsigma, \aleph=$ const. In particular, there could be a grid node $\left(x_{*}, t_{* *}\right)$ where the following inequality must be, but not valid:

$$
\varsigma^{\aleph} C_{r}^{2}+C_{r} \leq 1, \quad C_{r}=\frac{\Delta t \lambda_{\max }}{\Delta x}
$$

However, there is a possibility to take the parameters $\varkappa=\varkappa(x, t), \varsigma=\varsigma(x, t)$, and $\aleph=\aleph(x, t)$ such that the scheme COS2, (59), will be stable under (67). 
For instance, if we take $\varkappa, \varsigma, C_{r}=1, \aleph=0.5$ at $(x, t) \neq\left(x_{*}, t_{* *}\right)$ and $\aleph=0$ at $(x, t)=\left(x_{*}, t_{* *}\right)$, then (68) will be also valid.

In this subsection we will use analogous technic 8 to prove the stability of the developed nonstaggered central schemes. In view of Theorem 8 , the stability of the scheme MAC2, (64), will be investigated on the basis of its variational scheme. It is assumed that the bounded operator $\mathbf{A}(=\partial \mathbf{f}(\mathbf{u}) / \partial \mathbf{u})$ in (22) is Fréchet-differentiable on the set $\Omega_{\mathbf{u}} \subset \mathbb{R}^{M}$, and its derivative is bounded on $\Omega_{\mathbf{u}}$. Considering that $\mathbf{v}_{i}^{n}$ in (59) is Lipschitz-continuous, we write

$$
\left\|\mathbf{v}_{i+1}^{n}-\mathbf{v}_{i}^{n}\right\|_{2} \leq C_{v} \Delta x, \quad C_{v}=\text { const. }
$$

By virtue of (28), the second term in right-hand side of (64) can be written in the form

$$
\varkappa \frac{\Delta x}{8}\left(\mathbf{d}_{i+1}^{n}-\mathbf{d}_{i-1}^{n}\right)=\frac{\varkappa}{8}\left[\mathbb{B}_{i}^{n} \cdot\left(\mathbf{v}_{i+1}^{n}-\mathbf{v}_{i}^{n}\right)-\mathbb{A}_{i-1}^{n} \cdot\left(\mathbf{v}_{i}^{n}-\mathbf{v}_{i-1}^{n}\right)\right] .
$$

Then, the variational scheme corresponding to (64) is the following

$$
\begin{gathered}
\delta \mathbf{v}_{i}^{n+1}-0.25\left(\delta \mathbf{v}_{i+1}^{n}+2 \delta \mathbf{v}_{i}^{n}+\delta \mathbf{v}_{i-1}^{n}\right) \\
+\frac{\Delta t}{2 \Delta x}\left(\mathbf{A}_{i+1}^{n} \cdot \delta \mathbf{v}_{i+1}^{n}-\mathbf{A}_{i-1}^{n} \cdot \delta \mathbf{v}_{i-1}^{n}\right) \\
+\frac{\varkappa}{8}\left[\mathbb{B}_{i}^{n} \cdot\left(\delta \mathbf{v}_{i+1}^{n}-\delta \mathbf{v}_{i}^{n}\right)-\mathbb{A}_{i-1}^{n} \cdot\left(\delta \mathbf{v}_{i}^{n}-\delta \mathbf{v}_{i-1}^{n}\right)\right] \\
-\varsigma \frac{(\Delta t)^{2}}{2 \Delta x^{2}}\left[\mathbf{D}_{i+0.5}^{n} \cdot\left(\delta \mathbf{v}_{i+1}^{n}-\delta \mathbf{v}_{i}^{n}\right)-\mathbf{D}_{i-0.5}^{n} \cdot\left(\delta \mathbf{v}_{i}^{n}-\delta \mathbf{v}_{i-1}^{n}\right)\right]= \\
+\varsigma \frac{(\Delta t)^{2}}{2 \Delta x^{2}}\left[\left(\delta \mathbf{D}_{i+0.5}^{n}\right) \cdot\left(\mathbf{v}_{i+1}^{n}-\mathbf{v}_{i}^{n}\right)-\left(\delta \mathbf{D}_{i-0.5}^{n}\right) \cdot\left(\mathbf{v}_{i}^{n}-\mathbf{v}_{i-1}^{n}\right)\right] \\
-\frac{\varkappa}{8}\left[\left(\delta \mathbb{B}_{i}^{n}\right) \cdot\left(\mathbf{v}_{i+1}^{n}-\mathbf{v}_{i}^{n}\right)-\left(\delta \mathbb{A}_{i-1}^{n}\right) \cdot\left(\mathbf{v}_{i}^{n}-\mathbf{v}_{i-1}^{n}\right)\right] .
\end{gathered}
$$

By virtue of (42) and (23), we find

$$
\left\|\delta \mathbb{A}_{i}^{n}\right\|_{2},\left\|\delta \mathbb{B}_{i}^{n}\right\|_{2} \leq 4 \aleph, \quad\left\|\left(\mathbf{A}_{i}^{n}\right)^{2}\right\|_{2},\left\|\left(\mathbf{A}_{i+1}^{n}\right)^{2}\right\|_{2} \leq \lambda_{\max }^{2} .
$$

Thus, we may write that

$$
\left\|\delta \mathbf{D}_{i+0.5}^{n}\right\|_{2},\left\|\delta\left[\mathbf{D}_{i-0.5}^{n}\right]\right\|_{2} \leq 2 \lambda_{\max }^{2} .
$$

Then, by virtue of (21), (69), (72)-(73), and since $0 \leq \varkappa, \varsigma, \aleph \leq 1$, we find the following estimate for the right-hand side of (71):

$$
\begin{gathered}
\varsigma \frac{(\Delta t)^{2}}{2 \Delta x^{2}}\left\|\left(\delta \mathbf{D}_{i+0.5}^{n}\right) \cdot\left(\mathbf{v}_{i+1}^{n}-\mathbf{v}_{i}^{n}\right)-\left(\delta \mathbf{D}_{i-0.5}^{n}\right) \cdot\left(\mathbf{v}_{i}^{n}-\mathbf{v}_{i-1}^{n}\right)\right\|_{2} \\
\quad+\left\|\frac{\varkappa}{8}\left[\left(\delta \mathbb{B}_{i}^{n}\right) \cdot\left(\mathbf{v}_{i+1}^{n}-\mathbf{v}_{i}^{n}\right)-\left(\delta \mathbb{A}_{i-1}^{n}\right) \cdot\left(\mathbf{v}_{i}^{n}-\mathbf{v}_{i-1}^{n}\right)\right]\right\|_{2} \leq
\end{gathered}
$$




$$
\begin{gathered}
\varsigma \frac{(\Delta t)^{2}}{2 \Delta x^{2}}\left(\left\|\delta \mathbf{D}_{i+0.5}^{n}\right\|_{2}\left\|\mathbf{v}_{i+1}^{n}-\mathbf{v}_{i}^{n}\right\|_{2}+\left\|\delta \mathbf{D}_{i-0.5}^{n}\right\|_{2} \cdot\left\|\mathbf{v}_{i}^{n}-\mathbf{v}_{i-1}^{n}\right\|_{2}\right) \\
+\frac{1}{8}\left(\left\|\delta \mathbb{B}_{i}^{n}\right\|_{2} \cdot\left\|\mathbf{v}_{i+1}^{n}-\mathbf{v}_{i}^{n}\right\|_{2}+\left\|\delta \mathbb{A}_{i-1}^{n}\right\|_{2} \cdot\left\|\mathbf{v}_{i}^{n}-\mathbf{v}_{i-1}^{n}\right\|_{2}\right) \leq \\
\left(1+2 C_{r}^{2}\right) C_{v} \mu_{0} \Delta t, \quad C_{r}=\frac{\Delta t \lambda_{\max }}{\Delta x} .
\end{gathered}
$$

Since the uniform stability with respect to the initial data implies the stability of scheme [48, pp. 390-392] (see also [8, Sec. 5]), we conclude, in view of (74), that the scheme (71) will be stable if the following scheme (i.e. (71) without the right-hand side) will be stable

$$
\begin{aligned}
\delta \mathbf{v}_{i}^{n+1}= & 0.25\left(\delta \mathbf{v}_{i+1}^{n}+2 \delta \mathbf{v}_{i}^{n}+\delta \mathbf{v}_{i-1}^{n}\right)-\frac{\Delta t}{2 \Delta x}\left(\mathbf{A}_{i+1}^{n} \cdot \delta \mathbf{v}_{i+1}^{n}-\mathbf{A}_{i-1}^{n} \cdot \delta \mathbf{v}_{i-1}^{n}\right) \\
+ & \varsigma \frac{(\Delta t)^{2}}{2 \Delta x^{2}}\left[\mathbf{D}_{i+0.5}^{n} \cdot\left(\delta \mathbf{v}_{i+1}^{n}-\delta \mathbf{v}_{i}^{n}\right)-\mathbf{D}_{i-0.5}^{n} \cdot\left(\delta \mathbf{v}_{i}^{n}-\delta \mathbf{v}_{i-1}^{n}\right)\right] \\
& \quad-\frac{\varkappa}{8}\left[\mathbb{B}_{i}^{n} \cdot\left(\delta \mathbf{v}_{i+1}^{n}-\delta \mathbf{v}_{i}^{n}\right)-\mathbb{A}_{i-1}^{n} \cdot\left(\delta \mathbf{v}_{i}^{n}-\delta \mathbf{v}_{i-1}^{n}\right)\right] .
\end{aligned}
$$

Since the bounded operator $\mathbf{A}(=\partial \mathbf{f}(\mathbf{u}) / \partial \mathbf{u})$ in (22) is Fréchet-differentiable on the set $\Omega_{\mathbf{u}} \subset \mathbb{R}^{M}$, and its derivative is bounded on $\Omega_{\mathbf{u}}$, the operator $\mathbf{A}$ is Lipschitz. In view of (69), we can write that $\left\|(\Delta t / \Delta x)\left(\mathbf{A}_{i-1}^{n}-\mathbf{A}_{i+1}^{n}\right) \cdot \delta \mathbf{v}_{i}^{n}\right\| \leq$ $C_{a} \Delta t\left\|\delta \mathbf{v}_{i}^{n}\right\|, C_{a}=$ const. Thus, adding the term $(\Delta t / \Delta x)\left(\mathbf{A}_{i-1}^{n}-\mathbf{A}_{i+1}^{n}\right) \cdot$ $\delta \mathbf{v}_{i}^{n}$ to the right-hand side of (75) has no effect on the stability of (75). To investigate the stability of (75) we rewrite it in the following form, where the term $0.5(\Delta t / \Delta x)\left(\mathbf{A}_{i-1}^{n}-\mathbf{A}_{i+1}^{n}\right) \cdot \delta \mathbf{v}_{i}^{n}$ is added.

$$
\begin{gathered}
\delta \mathbf{v}_{i}^{n+1}=\left(0.25 \mathbf{I}-\mathbf{E}_{i, i-1}^{n}\right) \cdot \delta \mathbf{v}_{i-1}^{n} \\
+\left(0.5 \mathbf{I}+\mathbf{E}_{i, i-1}^{n}+\mathbf{E}_{i, i+1}^{n}\right) \cdot \delta \mathbf{v}_{i}^{n}+\left(0.25 \mathbf{I}-\mathbf{E}_{i, i+1}^{n}\right) \cdot \delta \mathbf{v}_{i+1}^{n},
\end{gathered}
$$

where

$$
\begin{aligned}
\mathbf{E}_{i, i-1}^{n} & =\frac{\varkappa}{8} \mathbb{A}_{i-1}^{n}-\varsigma \frac{(\Delta t)^{2}}{2 \Delta x^{2}} \mathbf{D}_{i-0.5}^{n}-\frac{\Delta t}{2 \Delta x} \mathbf{A}_{i-1}^{n}, \\
\mathbf{E}_{i, i+1}^{n} & =\frac{\varkappa}{8} \mathbb{B}_{i}^{n}-\varsigma \frac{(\Delta t)^{2}}{2 \Delta x^{2}} \mathbf{D}_{i+0.5}^{n}+\frac{\Delta t}{2 \Delta x} \mathbf{A}_{i+1}^{n} .
\end{aligned}
$$

We write, in view of (23) and (42), that the spectrum $s\left(\mathbf{E}_{i, j}^{n}\right) \subseteq\left[L_{\min , i}^{n}, L_{\max , i}^{n}\right]$, where $j=i \pm 1$ and

$$
L_{\min , i}^{n} \geq-0.5 \varsigma C_{r}^{2}-0.5 C_{r}, L_{\max , i}^{n} \leq 0.5 \varkappa \aleph-0.25 \varsigma C_{r}^{2}+0.5 C_{r} .
$$

Hence, by virtue of [7, Theorem 2.10] we find that the scheme (76) will be stable if

$$
\max _{\lambda \in\left[L_{\min , i}^{n}, L_{\max , i}^{n}\right]}(|0.25-\lambda|+|0.25+\lambda|) \leqslant 0.5, \quad \forall i, n .
$$


We obtain from (80) the following sufficient conditions for the stability of the variational scheme (71)

$$
\begin{gathered}
-0.5 \varsigma C_{r}^{2}-0.5 C_{r} \geq-0.25 \\
0.5 \varkappa \aleph-0.25 \varsigma C_{r}^{2}+0.5 C_{r} \leq 0.25, \quad C_{r}=\frac{\Delta t \lambda_{\max }}{\Delta x} .
\end{gathered}
$$

Let us note that the value of $L_{\max , i}^{n}$ in (79) can rarely be attained. It could be the case at the point $\left(x_{i}, t_{n}\right)$ only if $\left\|\mathbb{B}_{i}^{n}\right\|_{2}=4 \aleph,\left\|\mathbf{A}_{i+1}^{n}\right\|_{2}=\lambda_{\max }$, and $\left\|\mathbf{A}_{i}^{n}\right\|_{2}=0$ (or, what is the same, $\left\|\mathbb{A}_{i-1}^{n}\right\|_{2}=4 \aleph,\left\|\mathbf{A}_{i-1}^{n}\right\|_{2}=\lambda_{\max }$, and $\left\|\mathbf{A}_{i}^{n}\right\|_{2}=0$ ). Moreover, considering the modification of the scheme (76), where $\mathbf{D}_{i-0.5}^{n}$ is replaced by $\left(\mathbf{A}_{i-1}^{n}\right)^{2}$, namely

$$
\begin{aligned}
\mathbf{E}_{i, i-1}^{n} & =\frac{\varkappa}{8} \mathbb{A}_{i-1}^{n}-\varsigma \frac{(\Delta t)^{2}}{2 \Delta x^{2}}\left(\mathbf{A}_{i-1}^{n}\right)^{2}-\frac{\Delta t}{2 \Delta x} \mathbf{A}_{i-1}^{n}, \\
\mathbf{E}_{i, i+1}^{n} & =\frac{\varkappa}{8} \mathbb{B}_{i}^{n}-\varsigma \frac{(\Delta t)^{2}}{2 \Delta x^{2}}\left(\mathbf{A}_{i+1}^{n}\right)^{2}+\frac{\Delta t}{2 \Delta x} \mathbf{A}_{i+1}^{n},
\end{aligned}
$$

we note (see Proof in [7, Theorem 2.10]) that the stability of the modified scheme, (76), (83)-(84), implies the stability of the scheme (76)-(78), since the operator A is Lipschitz. Hence, instead of (82), we will consider less rigid requirement:

$$
0.5 \varkappa \aleph-0.5 \varsigma C_{r}^{2}+0.5 C_{r} \leq 0.25 \text {. }
$$

Let $\varsigma=1$, then, by virtue of (81) and (85), we find the stability condition for the variational scheme (71):

$$
C_{r} \leq 0.5(\sqrt{3}-1), \quad \varkappa \aleph \leq 2-\sqrt{3} .
$$

Thus, in view of Theorem 8 , the scheme MAC2, (64), will be stable if (86) will be valid.

Notice, if $\varsigma \rightarrow 0$ in (81) and (82), then we obtain the stability condition for the scheme MAC1, (50):

$$
\varkappa \aleph+C_{r} \leq 0.5, \quad C_{r}=\frac{\Delta t \lambda_{\max }}{\Delta x} .
$$

It is significant that the stability conditions (81), (85), and hence (86), is found by virtue of [7, Theorem 2.10] and, therefore, it is assumed that $\mathbf{E}_{i, j}^{n}$ is Lipschitz, i.e. $\mathbf{E}_{i, i+1}^{n}=\mathbf{E}_{i, i-1}^{n}+O(\Delta x)$. For the sake of brevity, we associate the Landau symbol $O(\Delta x)$ with Lipschitz-continuity of a grid function whose increment can be estimated in norm by a constant times $\Delta x$ for $\Delta x$ small enough. Note that $\mathbf{E}_{i, j}^{n}$ is Lipschitz if $\mathbf{A}_{i}^{n}$, $\mathbb{A}_{i}^{n}$, and $\mathbb{B}_{i}^{n}$ are Lipschitz. It is easy to see that $\mathbf{A}_{i}^{n}$ will be Lipschitz if $\mathbf{u}_{\epsilon}^{n}(x)$ (the solution to (65) at $t=t_{n}$ ) will be Lipschitzcontinuous. Actually, since the bounded operator $\mathbf{A}\left(\mathbf{u}_{\epsilon}\right)\left(=\left.(\partial \mathbf{f} / \partial \mathbf{u})\right|_{\mathbf{u}=\mathbf{u}_{\epsilon}}\right)$ is Fréchet-differentiable on the set $\Omega_{\mathbf{u}} \subset \mathbb{R}^{M}$, and its derivative is bounded on $\Omega_{\mathbf{u}}$, we write: $\left\|\mathbf{A}_{i+1}^{n}-\mathbf{A}_{i}^{n}\right\| \leq C_{A}\left\|\mathbf{u}_{\epsilon, i+1}^{n}-\mathbf{u}_{\epsilon, i}^{n}\right\| \leq C_{A} C_{u} \Delta x$, where $C_{A}, C_{u}$ 
$=$ const. We may guarantee that $\mathbb{A}_{i}^{n}$ and $\mathbb{B}_{i}^{n}$ will be Lipschitz if $\mathbf{u}_{\epsilon}^{n}(x)$ will be differentiable. Actually, we may write, by virtue of (28), that $\mathbb{A}_{i}=\mathbf{I}+O(\Delta x)$, and $\mathbb{B}_{i}=\mathbf{I}+O(\Delta x)$. Hence, Lipschitz-continuity of $\mathbb{A}_{i}^{n}$ and $\mathbb{B}_{i}^{n}$ is obvious. However, if $\mathbf{u}_{\epsilon}^{n}(x)$ is only Lipschitz-continuous, then we can not guarantee that $\mathbb{A}_{i}^{n}$ and $\mathbb{B}_{i}^{n}$ are also Lipschitz-continuous. In such a case a generalization of [7. Theorem 2.10] can be useful, namely the case when the scheme coefficients depend on several matrices. Then we obtain that (76), (83)-(84) will be stable if

$$
\max _{\lambda_{1}, \lambda_{2} \in\left[L_{\min , i}^{n}, L_{\text {max }, i}^{n}\right]}\left(\left|0.25-\lambda_{1}\right|+\left|0.25-\lambda_{2}\right|+\left|0.5+\lambda_{1}+\lambda_{2}\right|\right) \leqslant 1, \quad \forall i, n .
$$

By virtue of (88), we find that the scheme MAC2, (64), will be stable under the same conditions (81), 85), and, hence, under (86).

Let us note that the stability conditions, (866), are, in general, apt to be unduly rigid. Assuming that $\aleph=\aleph\left(x_{i}, t_{n}\right)$ as well as $C_{r}=C_{r}\left(t_{n}\right)$, and using Weyl's inequalities (see, e.g., 22, Chap. 3]), we can find less rigid stability conditions than (86). Let $\lambda_{i}^{n, k}$ denote the $k-t h$ singular value of $\mathbf{A}_{i}^{n}, \lambda_{\max , i}^{n}=$ $\max _{k} \lambda_{i}^{n, k}, \lambda_{\max }^{n}=\max _{i} \lambda_{\max , i}^{n}$, and let $\alpha_{i}^{n, k}$ denote the $k-t h$ singular value of $\mathbb{A}_{i}^{n}$, $\alpha_{\min , i}^{n}=\min _{k} \alpha_{i}^{n, k}$. Then, given the pre-assigned $\aleph=\widehat{\aleph} \equiv$ const and $C_{r}=\widehat{C}_{r} \equiv$ const $\leq 0.5$, we find the sought-after stability conditions at $t=t_{n}$, employing the following two-step procedure.

\section{Algorithm 10}

Step 1. Given $C_{r}=\widehat{C}_{r} \leq 0.5$ and $\tau^{n}=\Delta x \widehat{C}_{r} / \lambda_{\max }^{n}$, we, by virtue of Weyl's inequalities [22, Chap. 3], estimate $L_{\max , i}^{n}$ :

$$
L_{\max , i}^{n} \leq 0.5 \varkappa \aleph_{i}^{n}-0.5\left(\frac{\tau^{n}}{\Delta x}\right)^{2}\left(\lambda_{\max , i}^{n}\right)^{2}+0.5 \frac{\tau^{n}}{\Delta x} \lambda_{\max , i}^{n} .
$$

In view of (88) and (89), we find

$$
\aleph_{i}^{n}=\min \left[\widehat{\aleph},\left(\frac{\tau^{n}}{\Delta x}\right)^{2} \frac{\left(\lambda_{\max , i}^{n}\right)^{2}}{\varkappa}-\frac{\tau^{n}}{\varkappa \Delta x} \lambda_{\max , i}^{n}+\frac{1}{2 \varkappa}\right] .
$$

Step 2.Given $\aleph_{i}^{n}$, (90), we find the derivative $\mathbf{d}_{i}^{n}$ using (43) and, by virtue of (28), the diagonal matrix $\mathbb{A}_{i}^{n}$, and hence $\alpha_{\min , i}^{n}$. We, by virtue of Weyl's inequalities [22, Chap. 3], estimate $L_{\min , i}^{n}$ :

$$
L_{\min , i}^{n} \geq \frac{\varkappa \alpha_{\min , i}^{n}}{8}-0.5\left(\frac{\tau^{n}}{\Delta x}\right)^{2}\left(\lambda_{\max , i}^{n}\right)^{2}-0.5 \frac{\tau^{n}}{\Delta x} \lambda_{\max , i}^{n} .
$$

Let $\tau_{i}^{n}$ be used instead of $\tau^{n}$ in (91). In view of (88) and (91), the following inequality must be valid.

$$
\frac{\varkappa \alpha_{\min , i}^{n}}{4}-\left(\frac{\tau_{i}^{n}}{\Delta x}\right)^{2}\left(\lambda_{\max , i}^{n}\right)^{2}-\frac{\tau_{i}^{n}}{\Delta x} \lambda_{\max , i}^{n} \geq-0.5 .
$$


We obtain from (92):

$$
\tau_{i}^{n} \frac{\lambda_{\max , i}^{n}}{\Delta x} \leq \frac{\sqrt{3+\varkappa \alpha_{\min , i}^{n}}-1}{2}
$$

Thus, given $\tau^{n}\left(=\Delta x \widehat{C}_{r} / \lambda_{\max }^{n}\right)$ and $\tau_{i}^{n}$, (93), we find the time increment, $\Delta t^{n}$, at $t=t_{n}$ :

$$
\Delta t^{n}=\min \left(\tau^{n}, \min _{i} \tau_{i}^{n}\right) \Rightarrow C_{r}^{n}=\frac{\Delta t^{n} \lambda_{\max }^{n}}{\Delta x}
$$

\section{Operator splitting schemes}

By virtue of the operator-splitting idea [6], 13], [19], [32] (see also LOS in [48]), the following chain of equations corresponds to the problem (1)

$$
\begin{gathered}
\frac{1}{2} \frac{\partial \mathbf{U}}{\partial t}=\frac{1}{\tau} \mathbf{q}(\mathbf{U}), \quad t_{n}<t \leq t_{n+0.5}, \quad \mathbf{U}\left(\mathbf{x}, t_{n}\right)=\mathbf{U}^{n}(\mathbf{x}) \\
\frac{1}{2} \frac{\partial \mathbf{U}}{\partial t}+\sum_{j=1}^{N} \frac{\partial}{\partial x_{j}} \mathbf{f}_{j}(\mathbf{U})=0, t_{n+0.5}<t \leq t_{n+1}, \mathbf{U}\left(\mathbf{x}, t_{n+0.5}\right)=\mathbf{U}^{n+0.5}(\mathbf{x}),
\end{gathered}
$$

where $\mathbf{U}^{n}(\mathbf{x})$ denotes the solution to (96) at $t=t_{n}, \mathbf{U}^{n+0.5}(\mathbf{x})$ denotes the solution to (95) at $t=t_{n+0.5}$. If a high-resolution method is used directly for the homogeneous conservation law (96), then it is natural to use a high-order scheme for (95). As applied to, in general, stiff $(\tau \ll 1)$ System (11), the second order schemes can be constructed on the basis of operator-splitting techniques with ease if (95) will be approximated by an implicit scheme and (96) by an explicit one, see Proposition 4.2 in [7. As an example, let us develop a central scheme for a 1-D version of (11). After operator-splitting, the 1-D equation can be represented by the chain of equations, namely (95) and

$$
\frac{1}{2} \frac{\partial \mathbf{U}}{\partial t}+\frac{\partial}{\partial x} \mathbf{f}(\mathbf{U})=0, t_{n+0.5}<t \leq t_{n+1}, \mathbf{U}\left(x, t_{n+0.5}\right)=\mathbf{U}^{n+0.5}(x) .
$$

Let us first consider the case when the following first-order implicit scheme be used for (95)

$$
\mathbf{v}_{i}^{n+0.5}=\mathbf{v}_{i}^{n}+\frac{\Delta t}{\tau} \mathbf{q}\left(\mathbf{v}_{i}^{n+0.5}\right),
$$

and a central scheme with nonstaggered grid cells will be used for (97). We rewrite the scheme MAC1, (50), to read

$$
\begin{aligned}
\mathbf{v}_{i}^{n+1}= & 0.25\left(\mathbf{v}_{i-1}^{n+0.5}+2 \mathbf{v}_{i}^{n+0.5}+\mathbf{v}_{i+1}^{n+0.5}\right)-\varkappa \frac{\Delta x}{8}\left(\mathbf{d}_{i+1}^{n+0.5}-\mathbf{d}_{i-1}^{n+0.5}\right) \\
& -\frac{\Delta t}{2 \Delta x}\left(\mathbf{f}_{i+1}^{n+0.5}-\mathbf{f}_{i-1}^{n+0.5}\right), \quad \varkappa=\text { const }, 0 \leq \varkappa \leq 1,
\end{aligned}
$$


where $\mathbf{d}_{i}^{n+0.5}$ denotes the derivative of interpolant at $x=x_{i}$. It is clear that the scheme (99) approximates (97) with the accuracy $O\left(\Delta t+(\Delta x)^{2}\right)$, however, in view of Proposition 4.2 in [7, the scheme (98)-(99), taken as a whole, is of the second order approximation for the 1-D version of (1).

Let us develop another nonstaggered central scheme approximating a 1-D version of (11) with the accuracy $O\left((\Delta t)^{2}+(\Delta x)^{2}\right)$ and such that its components (after operator splitting) will be of the second order. It can be done on the basis of the second order scheme (98), (99) with ease. Notice, adding to and subtracting from Equation (4.10) in [7] (rewritten for $t_{n}<t \leq t_{n+1}$ ) the same quantity is equivalent to adding this quantity to (99) and subtracting it from (98). Let $0.125(\Delta t)^{2}\left(\partial^{2} \mathbf{U} / \partial t^{2}\right)_{i}^{n+0.5}$ be this quantity, then we obtain the following scheme, instead of (98), (99),

$$
\begin{gathered}
\mathbf{v}_{i}^{n+0.5}=\mathbf{v}_{i}^{n}+\frac{\Delta t}{\tau} \mathbf{q}_{i}^{n+0.5}-\frac{(\Delta t)^{2}}{8}\left(\frac{\partial^{2} \mathbf{U}}{\partial t^{2}}\right)_{i}^{n+0.5}, \\
\mathbf{v}_{i}^{n+1}=0.25\left(\mathbf{v}_{i-1}^{n+0.5}+2 \mathbf{v}_{i}^{n+0.5}+\mathbf{v}_{i+1}^{n+0.5}\right)-\varkappa \frac{\Delta x}{8}\left(\mathbf{d}_{i+1}^{n+0.5}-\mathbf{d}_{i-1}^{n+0.5}\right) \\
-\frac{\Delta t}{2 \Delta x}\left(\mathbf{f}_{i+1}^{n+0.5}-\mathbf{f}_{i-1}^{n+0.5}\right)+\frac{(\Delta t)^{2}}{8}\left(\frac{\partial^{2} \mathbf{U}}{\partial t^{2}}\right)_{i}^{n+0.5} .
\end{gathered}
$$

Thus, the scheme (100) as well as the scheme (101) are of the second order, and the scheme (100)-(101), taken as a whole, is of the second order as well. Using Taylor series expansion, and central differencing, we find

$$
\begin{gathered}
\mathbf{v}_{i}^{n+0.25}=\mathbf{v}_{i}^{n+0.5}-\frac{\Delta t}{4}\left(\frac{\partial \mathbf{U}}{\partial t}\right)_{i}^{n+0.5}+ \\
\frac{1}{2}\left(\frac{\Delta t}{4}\right)^{2}\left(\frac{\partial^{2} \mathbf{U}}{\partial t^{2}}\right)_{i}^{n+0.5}+O\left((\Delta t)^{3}\right), \\
\mathbf{v}_{i}^{n+0.5}=\mathbf{v}_{i}^{n}+\frac{\Delta t}{2}\left(\frac{\partial \mathbf{U}}{\partial t}\right)_{i}^{n+0.25}+O\left((\Delta t)^{3}\right) .
\end{gathered}
$$

We obtain, by virtue of (62), (97), that

$$
\frac{\partial^{2} \mathbf{U}}{\partial t^{2}}=-2 \frac{\partial}{\partial t}\left(\frac{\partial \mathbf{f}}{\partial x}\right)=-2 \frac{\partial}{\partial x}\left(\frac{\partial \mathbf{f}}{\partial t}\right)=4 \frac{\partial}{\partial x}\left(\mathbf{A}^{2} \cdot \frac{\partial \mathbf{U}}{\partial x}\right),
$$

where $\mathbf{A}=\partial \mathbf{f} / \partial \mathbf{U}$. Then

$$
\begin{gathered}
{\left[\frac{\partial}{\partial x}\left(\mathbf{A}^{2} \cdot \frac{\partial \mathbf{U}}{\partial x}\right)\right]_{i}^{n+0.5}=\frac{1}{\Delta x}\left[\mathbf{D}_{i+0.5}^{n+0.5} \cdot \frac{\mathbf{v}_{i+1}^{n+0.5}-\mathbf{v}_{i}^{n+0.5}}{\Delta x}\right.} \\
\left.-\mathbf{D}_{i-0.5}^{n+0.5} \cdot \frac{\mathbf{v}_{i}^{n+0.5}-\mathbf{v}_{i-1}^{n+0.5}}{\Delta x}\right]+O\left((\Delta x)^{2}\right)
\end{gathered}
$$


where $\mathbf{D}_{i+0.5}^{n+0.5}=0.5\left(\left(\mathbf{A}_{i+1}^{n+0.5}\right)^{2}+\left(\mathbf{A}_{i}^{n+0.5}\right)^{2}\right)$. By virtue of (95), (102)-(105), we rewrite Scheme (100)-101) to read

$$
\begin{gathered}
\mathbf{v}_{i}^{n+0.25}=\mathbf{v}_{i}^{n+0.5}-\frac{\Delta t}{4 \tau}\left(\mathbf{q}_{i}^{n+0.25}+\mathbf{q}_{i}^{n+0.5}\right) \\
\mathbf{v}_{i}^{n+0.5}=\mathbf{v}_{i}^{n}+\frac{\Delta t}{\tau} \mathbf{q}_{i}^{n+0.25} \\
\mathbf{v}_{i}^{n+1}=0.25\left(\mathbf{v}_{i-1}^{n+0.5}+2 \mathbf{v}_{i}^{n+0.5}+\mathbf{v}_{i+1}^{n+0.5}\right)-\varkappa \frac{\Delta x}{8}\left(\mathbf{d}_{i+1}^{n+0.5}-\mathbf{d}_{i-1}^{n+0.5}\right) \\
+\frac{\varsigma(\Delta t)^{2}}{2(\Delta x)^{2}}\left[\mathbf{D}_{i+0.5}^{n+0.5} \cdot\left(\mathbf{v}_{i+1}^{n+0.5}-\mathbf{v}_{i}^{n+0.5}\right)-\mathbf{D}_{i-0.5}^{n+0.5} \cdot\left(\mathbf{v}_{i}^{n+0.5}-\mathbf{v}_{i-1}^{n+0.5}\right)\right] \\
-\frac{\Delta t}{2 \Delta x}\left(\mathbf{f}_{i+1}^{n+0.5}-\mathbf{f}_{i-1}^{n+0.5}\right) .
\end{gathered}
$$

If $\varsigma=0$, then (108) coincides with (99), being $O\left(\Delta t+(\Delta x)^{2}\right)$ accurate. If $\varkappa=1$ and $\varsigma=1$, then Scheme (106)-(108) approximates the 1-D version of (1) with the accuracy $O\left((\Delta t)^{2}+(\Delta x)^{2}\right)$. The scheme (108) coincides, in fact, with the scheme MAC2, (64), and hence the scheme (108) will be stable if (86) will be valid. Notice, the less rigid conditions for the stability of the scheme (108) can be found by means of Algorithm [10.

Let us note that in practice (e.g., 32, 48]) the operator-splitting techniques find a wide range of application in designing economical schemes for Eq. (96) in the domains of complicated geometry. The resulting method, in general, will be only first-order accurate in time because of the splitting [32, 48]. Thus, in line with established practice we will replace the multidimensional Eq. (96) by the chain of the one-dimensional equations:

$$
\frac{1}{2 N} \frac{\partial \mathbf{U}_{j}}{\partial t}+\frac{\partial}{\partial x_{j}} \mathbf{f}_{j}\left(\mathbf{U}_{j}\right)=0, t_{n+0.5+(j-1) /(2 N)}<t \leq t_{n+0.5+j /(2 N)}
$$

where $\mathbf{U}_{j}\left(x, t_{n+0.5+(j-1) /(2 N)}\right)=\mathbf{U}_{j-1}\left(x, t_{n+0.5+(j-1) /(2 N)}\right), j=1,2, \ldots N$, $\mathbf{U}_{0}\left(x, t_{n+0.5}\right)$ denotes the solution to (95) at $t=t_{n+0.5}$. Eq. (95) will be approximated by the first-order implicit scheme, (98), or a second-order implicit Runge-Kutta scheme. In particular, it can be used (106)-(107) or the following implicit Runge-Kutta scheme

$$
\mathbf{v}_{i}^{n+0.25}=\mathbf{v}_{i}^{n+0.5}-\frac{\Delta t}{2 \tau} \mathbf{q}_{i}^{n+0.5}, \mathbf{v}_{i}^{n+0.5}=\mathbf{v}_{i}^{n}+\frac{\Delta t}{\tau} \mathbf{q}_{i}^{n+0.25},
$$

since these schemes possess a discrete analogy to the continuous asymptotic limit (see, e.g., 24], 37, 41]). Let us note that the scheme (110) is an implicit analogue to the well known Runge-Kutta scheme, which is referred, originally due to Runge, as modified Euler method [20]:

$$
\mathbf{v}_{i}^{n+0.25}=\mathbf{v}_{i}^{n}+\frac{\Delta t}{2 \tau} \mathbf{q}_{i}^{n}, \mathbf{v}_{i}^{n+0.5}=\mathbf{v}_{i}^{n}+\frac{\Delta t}{\tau} \mathbf{q}_{i}^{n+0.25} .
$$




\section{$5 \quad$ Examples}

In this section, we are mainly concerned with verification of the second order central schemes COS2, (59) and MAC2, (64), as well as the splitting schemes (106)-(108), and (109).

\subsection{Scalar non-linear equation}

As the first stage in the verification, we will focus on the following scalar 1-D version of the problem (1):

$$
\frac{\partial u}{\partial t}+\frac{\partial}{\partial x} f(u)=0, \quad x \in \mathbb{R}, 0<t \leq T_{\max } ;\left.\quad u(x, t)\right|_{t=0}=u^{0}(x) .
$$

Let us first compare the schemes MAC2, (64), and COS2, (59), and demonstrate that these schemes are of the second order. We consider the linear transport equation, i.e. (112) with $f(u) \equiv u$, subject to the initial data: $u^{0}(x)=\sin (\pi x)$. The numerical solutions were computed under $\varkappa=\varsigma=1$. The scheme COS2, (59), will be stable, in view of (67), (68), if we take the CFL number $C r=\sqrt{3}-1$, and $\aleph=2-\sqrt{3}$. The scheme MAC2, (64), will be stable, in view of (86), under $C r=0.5(\sqrt{3}-1)$ and $\aleph=2-\sqrt{3}$. We will also verify the scheme COS1, (45), under $C r=0.5$ and $\aleph=0.25$, as well as the scheme MAC1, (50), under $C r=0.25$ and $\aleph=0.25$. Notice, in the case of schemes COS2 and COS1 the CFL numbers are two times higher than in the case of schemes MAC2 and MAC1, respectively. The reason is that the staggered schemes, COS2 and COS1, are solved twice during the time increment, $\Delta t$, in contrast to the nonstaggered schemes MAC2 and MAC1. $L_{1}$ errors, at $t=10,0 \leq x \leq 2$, versus the number of nodes are depicted in Table1.

Table 1: $L_{1}$ errors versus the number of nodes $(N)$

\begin{tabular}{|c|c|c|c|c|c|}
\hline$N$ & 1280 & 640 & 320 & 160 & 80 \\
\hline COS1 & $2.4 \times 10^{-3}$ & $4.9 \times 10^{-3}$ & $9.8 \times 10^{-3}$ & $2.0 \times 10^{-2}$ & $3.9 \times 10^{-2}$ \\
\hline COS2 & $1.5 \times 10^{-5}$ & $6.2 \times 10^{-5}$ & $2.5 \times 10^{-4}$ & $1.0 \times 10^{-3}$ & $3.9 \times 10^{-3}$ \\
\hline MAC1 & $2.5 \times 10^{-3}$ & $4.9 \times 10^{-3}$ & $1.0 \times 10^{-2}$ & $2.1 \times 10^{-2}$ & $4.3 \times 10^{-2}$ \\
\hline MAC2 & $5.6 \times 10^{-5}$ & $2.4 \times 10^{-4}$ & $9.8 \times 10^{-4}$ & $3.9 \times 10^{-3}$ & $1.5 \times 10^{-2}$ \\
\hline
\end{tabular}

Note (Table 1) that $L_{1}$ errors in the case of the scheme COS2 are approximately coincide with those appeared in the case of the scheme MAC2 under the double number of nodes. It is associated with the fact that in the case of the staggered scheme COS2, (59), the space increment is, actually, two times less than in the case of the nonstaggered scheme MAC2, (64).

Now we will solve the inviscid Burgers equation (i.e. $\left.f(u) \equiv u^{2} / 2\right)$ with the following initial condition

$$
u(x, 0)=\left\{\begin{array}{cc}
u_{0}, & x \in\left(h_{L}, h_{R}\right) \\
0, & x \notin\left(h_{L}, h_{R}\right)
\end{array} \quad, \quad h_{R}>h_{L}, u_{0}=\text { const } \neq 0 .\right.
$$


The exact solution to (112), (113) is given by

$$
u(x, t)=\left\{\begin{array}{lc}
u_{1}(x, t), & 0<t \leq T \\
u_{2}(x, t), & t>T
\end{array}\right.
$$

where $T=2 S / u_{0}, S=h_{R}-h_{L}$,

$$
\begin{gathered}
u_{1}(x, t)=\left\{\begin{array}{cc}
\frac{x-h_{L}}{b-h_{L}} u_{0}, & h_{L}<x \leq b, b=u_{0} t+h_{L} \\
u_{0}, & b<x \leq 0.5 u_{0} t+h_{R} \\
0, & x \leq h_{L} \text { or } x>0.5 u_{0} t+h_{R}
\end{array},\right. \\
u_{2}(x, t)=\left\{\begin{array}{cc}
\frac{2 S\left(x-h_{L}\right)}{\left(L-h_{L}\right)^{2}} u_{0}, & h_{L}<x \leq L \\
0, & x \leq h_{L} \text { or } x>L
\end{array},\right. \\
L=2 \sqrt{S^{2}+0.5 u_{0} S(t-T)}+h_{L} .
\end{gathered}
$$

First, it will be used the first order in time schemes MAC1, (50), and COS1, (45). The numerical solutions were computed on a uniform grid with spatial increments of $\Delta x=0.01$, the velocity $u_{0}=1$ in (113), $h_{L}=0.2, h_{R}=1$. In view of the stability condition, (87), for the scheme MAC1, we take the CFL number $C r=0.25$, the monotonicity parameter $\aleph=0.25$, and the parameter $\varkappa=1$. Having regard to the stability condition (67) under $\varsigma=0$, we verify the scheme COS1, (45), under $C r=0.5, \aleph=0.25$, and $\varkappa=1$. The results of simulations are depicted with the exact solution in Figure 2.
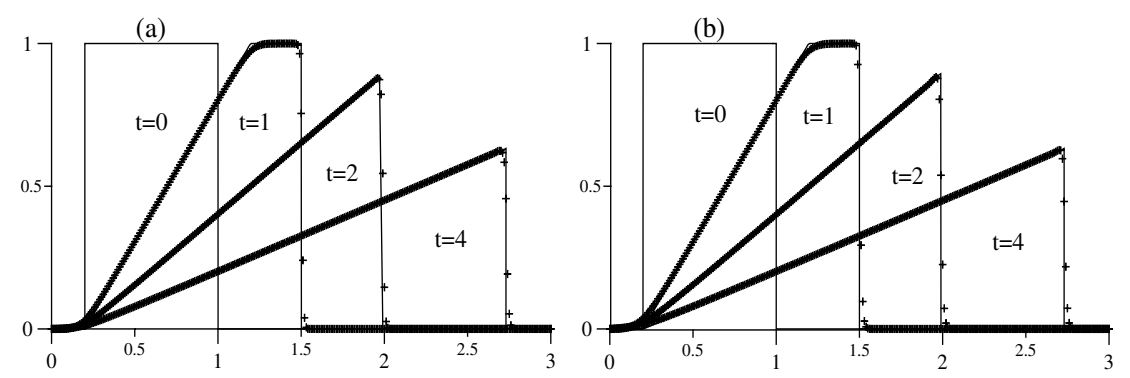

Figure 2: Inviscid Burgers equation. The schemes COS1 (a) at given $C_{r}=0.5$ and MAC1 (b) at given $C_{r}=0.25$ versus the analytical solution. Crosses: numerical solution; Solid line: analytical solution and initial data. $\Delta x=0.01$, $\aleph=0.25$.

We note (Figure 22) that the schemes COS1, (45), and MAC1, (50), exhibit similar results in spite of the fact that in the case of the scheme COS1 the space increments are, virtually, two times less than those in the case of MAC1. It should also be mentioned the absence of spurious oscillations in the numerical solutions (see Figure 2). However, if we take $\aleph=0.5$, then the spurious oscillations can be produced by the scheme COS1 (see [8, p. 2804]). Using Algorithm 10] at given $\widehat{\aleph}=0.5$, in the case of scheme MAC1 we obtain very slight but 
significant spurious oscillations, which indicate that the boundary maximum principle is violated. For instance, the maximum positive value of the dependent variable, $v$, at $t=1$ is $0.4 \%$ above the maximum value of $v$ at the boundary $t=0$. These spurious oscillations can be eradicated by decreasing the parameter $\varkappa$. Particularly, the spurious oscillations disappear if $\varkappa=0.7$, however, this introduces an additional numerical smearing. The results of simulations are not depicted here.

To test the schemes MAC2, (64), and COS2, (59), the inviscid Burgers equation was solved under the initial condition (113). The numerical solutions were computed under the same values of parameters as in the case of the schemes MAC1 and COS1, but $C_{r}$ and $\aleph$. In view of the stability condition, (86), for the scheme MAC2, we take the CFL number $C r=0.5(\sqrt{3}-1)$. It is also used Algorithm 10 at given $\widehat{\aleph}=0.5$. The scheme COS2 will be stable, in view of (67), (68), if we take $C r=\sqrt{3}-1$ and $\aleph=0.5$. The results of simulation are depicted with the exact solution in Figure 3 .
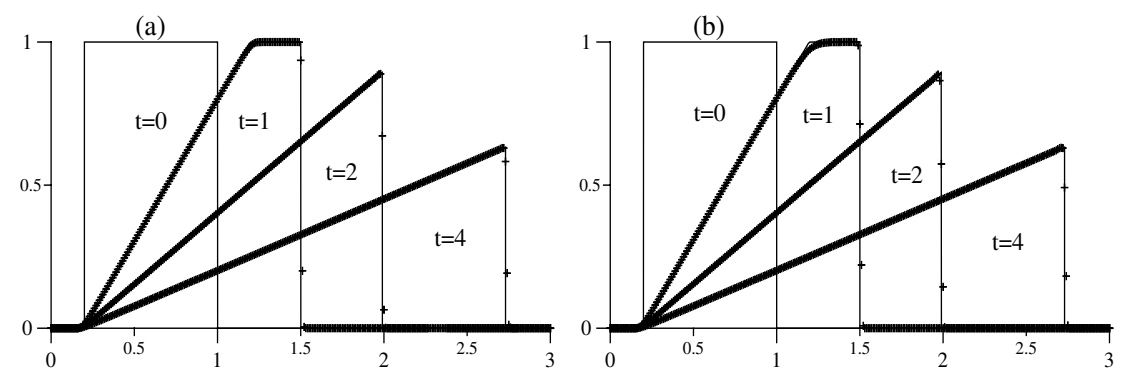

Figure 3: Inviscid Burgers equation. The schemes COS2 (a) at given $C_{r}=$ $\sqrt{3}-1$ and MAC2 (b) at given $C_{r}=0.5(\sqrt{3}-1)$ versus the analytical solution. Crosses: numerical solution; Solid line: analytical solution and initial data. $\Delta x=0.01, \aleph=\widehat{\aleph}=0.5$.

We note (Figure 3) that the schemes COS2 and MAC2 exhibit a typical second-order nature without any spurious oscillations. The scheme MAC2 exhibits more numerical smearing than the scheme COS2. Such a phenomenon has, at least, two reasons. First, in the case of the staggered scheme COS2 the space increment is, in fact, two times less than in the case of the nonstaggered scheme MAC2. Second place, using Algorithm 10 at given $\widehat{\aleph}=0.5$ we, in general, obtain $\aleph_{i}^{n}<\widehat{\aleph}$ at the grid node $\left(x_{i}, t_{n}\right)$, resulting in an additional numerical smearing.

\subsection{Hyperbolic conservation laws with relaxation}

Let us consider the model system of hyperbolic conservation laws with relaxation developed in [45]:

$$
\frac{\partial w}{\partial t}+\frac{\partial}{\partial x}\left(\frac{1}{2} u^{2}+a w\right)=0,
$$




$$
\frac{\partial z}{\partial t}+\frac{\partial}{\partial x} a z=\frac{1}{\tau} Q(w, z)
$$

where

$$
Q(w, z)=z-m\left(u-u_{0}\right), \quad u=w-q_{0} z,
$$

$\tau$ denotes the relaxation time of the system, $q_{0}, m, a$, and $u_{0}$ are constants. The Jacobian, A, can be written in the form

$$
\mathbf{A}=\left\{\begin{array}{cc}
w-q_{0} z+a & -q_{0}\left(w-q_{0} z\right) \\
0 & a
\end{array}\right\} .
$$

The system (118)-(119) has the following frozen [45] characteristic speeds $\lambda_{1}=$ $a, \lambda_{2}=u+a$. The equilibrium equation for (118)-(119) is

$$
\frac{\partial w}{\partial t}+\frac{\partial}{\partial x}\left(\frac{1}{2} u_{*}^{2}+a w\right)=0
$$

where

$$
u_{*}=w-q_{0} z_{*}, \quad z_{*}=\frac{m}{1+m q_{0}}\left(w-u_{0}\right) .
$$

The equilibrium characteristic speed $\lambda_{*}$ can be written in the form

$$
\lambda_{*}(w)=\frac{u_{*}(w)}{1+m q_{0}}+a .
$$

Pember's rarefaction test problem is to find the solution $\{w, z\}$ to (118)(119), and hence the function $u=u(x, t)$, under $\tau \rightarrow 0$, and where

$$
\begin{gathered}
\{w, z\}=\left\{\begin{array}{ll}
\left\{w_{L}, z_{*}\left(w_{L}\right)\right\}, & x<x_{0} \\
\left\{w_{R}, z_{*}\left(w_{R}\right)\right\}, & x>x_{0}
\end{array},\right. \\
0<u_{L}=w_{L}-q_{0} z_{*}\left(w_{L}\right)<u_{R}=w_{R}-q_{0} z_{*}\left(w_{R}\right) .
\end{gathered}
$$

The analytical solution of this problem can be found in 45. The parameters of the model system are assumed as follows: $q_{0}=-1, m=-1, u_{0}=3, a=1$, $\tau=10^{-8}$. The initial conditions of the rarefaction problem are defined by

$$
\begin{gathered}
u_{L}=2, \Longrightarrow z_{L}=m\left(u_{L}-u_{0}\right)=1, w_{L}=u_{L}+q_{0} z_{L}=1, \\
u_{R}=3, \Longrightarrow z_{R}=m\left(u_{R}-u_{0}\right)=0, w_{R}=u_{R}+q_{0} z_{R}=3 .
\end{gathered}
$$

The position of the initial discontinuity, $x_{0}$, is set according to the value of $a$ so that the solutions of all the rarefaction problems are identical 45]. Let a position, $x_{R}^{t}$, of leading edge or a position, $x_{L}^{t}$, of trailing edge of the rarefaction be known (e.g., $x_{R}^{t}=0.85, x_{L}^{t}=0.7$ in [45]), then

$$
x_{0}=x_{R}^{t}-\left(\frac{u_{R}}{1+m q_{0}}+a\right) t=x_{L}^{t}-\left(\frac{u_{L}}{1+m q_{0}}+a\right) t .
$$


At $t=0.3$, under (127)-(128) we have [45]

$$
u=\left\{\begin{array}{cc}
2, & x \leq 0.7 \\
2+\frac{x-0.7}{0.85-0.7}, & 0.7<x<0.85 \\
3, & x \geq 0.85
\end{array} .\right.
$$

The results of simulations, based upon the schemes MAC2, (64), and COS2, (59), together with (110), under different values of a grid spacing $\left(\Delta x=10^{-3}\right.$, $5 \times 10^{-4}$ ) are depicted in Figure 4. In view of the stability condition, (86), for the scheme MAC2, we take the CFL number $C r=0.5(\sqrt{3}-1)$. It is also used Algorithm 10 at given $\widehat{\aleph}=0.5$. The scheme COS2 will be stable, in view of (67), (68), if we take $C r=\sqrt{3}-1$ and $\aleph=0.5$.
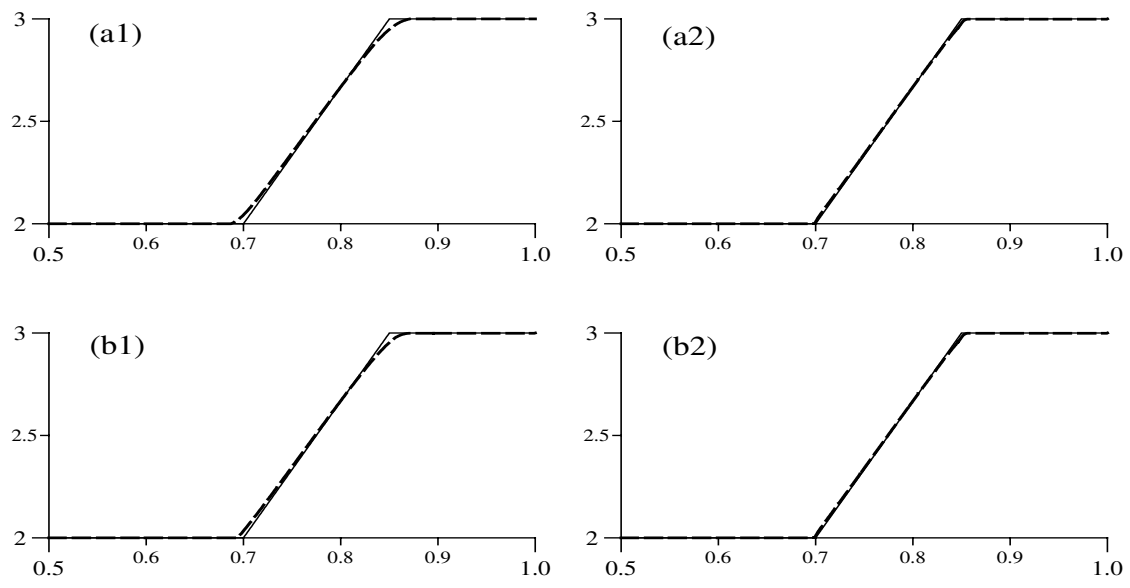

Figure 4: Pember's rarefaction test problem. The schemes COS2 (a1, a2) at given $C_{r}=\sqrt{3}-1$ and MAC2 $(\mathrm{b} 1, \mathrm{~b} 2)$ at given $C_{r}=0.5(\sqrt{3}-1)$ versus the analytical solution for $u$. Dashed line: numerical solution; Solid line: analytical solution. Time $t=0.3, \aleph=\widehat{\aleph}=0.5,(\mathrm{a} 1, \mathrm{~b} 1): \Delta x=0.001,(\mathrm{a} 2, \mathrm{~b} 2): \Delta x=$ 0.0005 .

One can clearly see (Figure 4) that the schemes MAC2 together with the Runge-Kutta scheme (110) as well as COS2 together with (110) are free from spurious oscillations. Recall that in the case of the staggered scheme COS2 the space increment is, in fact, two times less than in the case of the nonstaggered scheme MAC2. Moreover, using Algorithm 10 in the case of scheme MAC2, we, in general, obtain $\aleph_{i}^{n}<\widehat{\aleph}$ at the grid node $\left(x_{i}, t_{n}\right)$, resulting in an additional numerical smearing. Nevertheless, the scheme MAC2 can exhibit (Figure 4) even less numerical viscosity than the scheme COS2. 


\subsection{1-D Euler equation of gas dynamics}

In this subsection we apply the second order schemes COS2, (59), and MAC2, (64), to the Euler equations of gamma-law gas:

$$
\begin{gathered}
\frac{\partial \mathbf{u}(x, t)}{\partial t}+\frac{\partial}{\partial x} \mathbf{F}(\mathbf{u})=0, \quad x \in \mathbb{R}, t>0 ; \quad \mathbf{u}(x, 0)=\mathbf{u}^{0}(x), \\
\mathbf{u} \equiv\left\{u_{1}, u_{2}, u_{3}\right\}^{T}=\{\rho, \rho v, e\}^{T}, \quad \mathbf{F}(\mathbf{u})=\left\{\rho v, \rho v^{2}+p,(e+p) v\right\}^{T}, \\
e=\frac{p}{\gamma-1}+\frac{1}{2} \rho v^{2}, \quad \gamma=\text { const },
\end{gathered}
$$

where $\rho, v, p, e$ denote the density, velocity, pressure, and total energy respectively. We consider the Riemann problem subject to Riemann initial data

$$
\mathbf{u}^{0}(x)=\left\{\begin{array}{ll}
\mathbf{u}_{L} & x<x_{0} \\
\mathbf{u}_{R} & x>x_{0}
\end{array} \quad, \quad \mathbf{u}_{L}, \mathbf{u}_{R}=\text { const } .\right.
$$

The analytic solution to the Riemann problem can be found in [32, Sec. 14].

We solve the shock tube problem (see, e.g., [5], 23], 32, 33]) with Sod's initial data:

$$
\mathbf{u}_{L}=\left\{\begin{array}{c}
1 \\
0 \\
2.5
\end{array}\right\}, \quad \mathbf{u}_{R}=\left\{\begin{array}{c}
0.125 \\
0 \\
0.25
\end{array}\right\} .
$$

Following Balaguer and Conde [5] as well as Liu and Tadmor 33. we assume that the computational domain is $0 \leq x \leq 1$; the point $x_{0}$ is located at the middle of the interval $[0,1]$, i.e. $x_{0}=0.5$; the equations (131) are integrated up to $t=0.16$ on a spatial grid with 200 nodes as in [5] and in 33. Aiming to compare the schemes COS2 and MAC2, we, in view of the stability conditions (67), (68), and (86), take the CFL number $\mathrm{Cr}=\sqrt{3}-1$ (the scheme COS2), and $C r=0.5(\sqrt{3}-1)$ (the scheme MAC2). In both cases we take $\varkappa \aleph=2-\sqrt{3}$, where $\varkappa=0.94$. The reason why the parameter $\varkappa$ has to be less than unity is discussed in Section 3.2 (see also [8, Sec. 4]). The results of simulations are depicted in Figure 5 (left column) and Figure 6 (left column).

The results using the scheme COS2 (Figure 5. left column) are not worse in comparison to the corresponding third-order central results of [33, p. 418] as well as to the results obtained by the fourth-order non-oscillatory scheme in [5, p. 472]. The simulations in [5] and [33] were done under $\Delta t=0.1 \Delta x$ (i.e. $0.13 \lesssim C r \lesssim 0.22$ ). The fourth-order scheme [5, p. 472] gives a better resolution but, in contrast to the scheme COS2 (Figure 5. left column), can produce spurious oscillations. Let us note that the second-order scheme COS2 can give results analogous to those obtained by the fourth-order scheme [5, p. 472]. Namely, the higher will be the values of $\varkappa$ and $\aleph$, the better will be the resolution (see, e.g., Figure 5, right column). However, if $\varkappa=1$, then the scheme COS2 will be oscillatory [8, p. 2816]. It is apparent that $\varkappa$ should be reasonably less than unity to suppress spurious oscillations, while it should be as close to unity as possible to lose less in the order of accuracy of the scheme [8, 

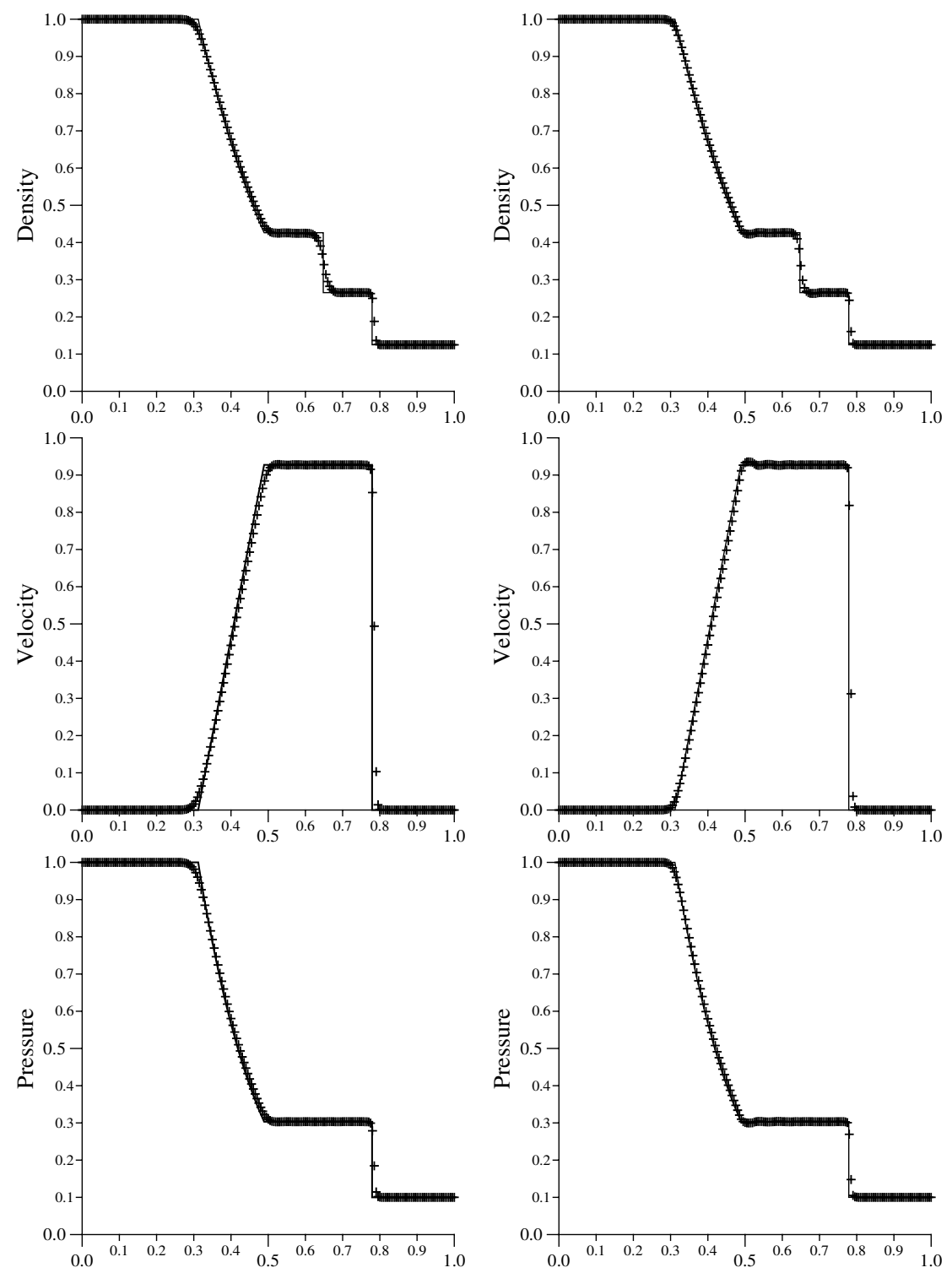

Figure 5: Sod's problem. Time $t=0.16$. The scheme COS2 versus the analytical solution. $\varkappa \aleph=2-\sqrt{3}, \varkappa=0.94$ (left column) and $\aleph=0.35, \varkappa=1$ (right column). Crosses: numerical solution; Solid line: analytical solution. $C_{r}=$ $\sqrt{3}-1, \Delta x=0.005$ 

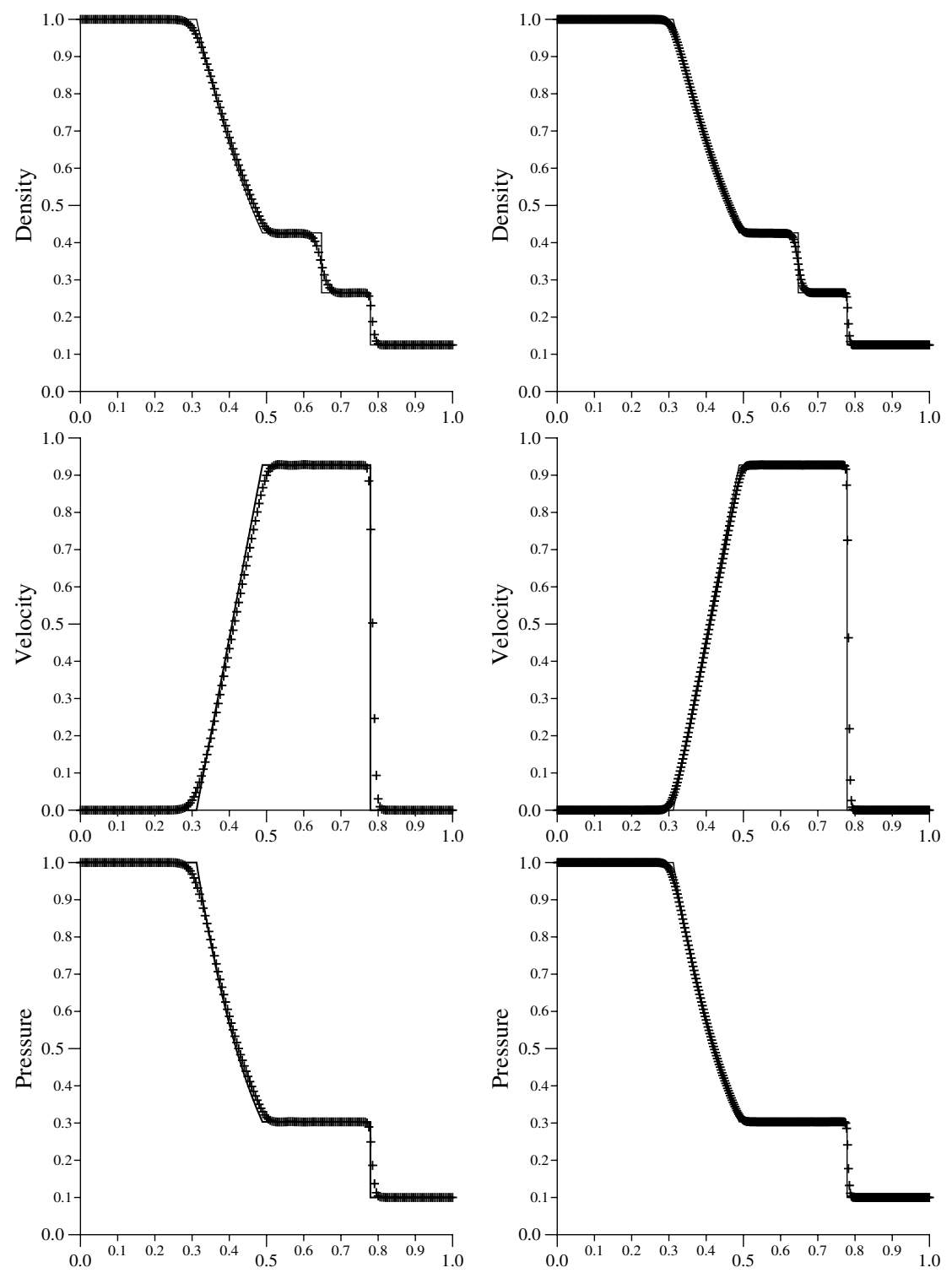

Figure 6: Sod's problem. The scheme MAC2 versus the analytical solution. Time $t=0.16 . C_{r}=0.5(\sqrt{3}-1), \varkappa \aleph=2-\sqrt{3}, \varkappa=0.94$. Crosses: numerical solution; Solid line: analytical solution. $\Delta x=0.005$ (left column), $\Delta x=0.0025$ (right column). 
p. 2816]. The results of using the scheme MAC2 (Figure 6, left column) are not worse in comparison to the corresponding second-order central results of [33, p. $418]$, in spite of the fact in the case of staggered scheme the space increment is, in effect, two times less than in the case of nonstaggered scheme. For the sake of illustration, the results using the scheme MAC2 under $\Delta x=0.0025$ are depicted in Figure 6 (right column). It is easily seen that the scheme MAC2 gives a better resolution than the scheme COS2 (Figure 5, left column).

\subsection{3-D axial symmetric gas dynamics}

We consider an adiabatic expansion of a vapor-plasma plume, produced by a laser beam on a target plane surface, into vacuum 3], i.e., the so called Anisimov's problem. It is assumed [3] that the expansion of the plasma plume may be described by the ideal gas dynamic equations with a constant adiabatic exponent $\gamma$. Let the target surface be perpendicular to the axis $z$ and located at $z=0$. Taking into account the symmetry of the plume with respect to the axis $z$, the gas-dynamic equations can be written as follows.

$$
\begin{gathered}
\frac{\partial \rho}{\partial t}+\frac{1}{r} \frac{\partial\left(r \rho v_{r}\right)}{\partial r}+\frac{\partial\left(\rho v_{z}\right)}{\partial z}=0 \\
\frac{\partial}{\partial t}\left(\rho v_{r}\right)+\frac{1}{r} \frac{\partial}{\partial r}\left[r \rho\left(v_{r}\right)^{2}\right]+\frac{\partial}{\partial z}\left(\rho v_{z} v_{r}\right)+\frac{\partial p}{\partial r}=0 \\
\frac{\partial}{\partial t}\left(\rho v_{z}\right)+\frac{\partial}{\partial z}\left[\rho\left(v_{z}\right)^{2}\right]+\frac{1}{r} \frac{\partial}{\partial r}\left(r \rho v_{z} v_{r}\right)+\frac{\partial p}{\partial z}=0 \\
\frac{\partial \rho E}{\partial t}+\frac{1}{r} \frac{\partial}{\partial r}\left[r v_{r}(\rho E+p)\right]+\frac{\partial}{\partial z}\left[v_{z}(\rho E+p)\right]=0 \\
\rho E=\frac{p}{\gamma-1}+0.5 \rho v^{2}, v^{2}=v_{r}^{2}+v_{z}^{2}, \gamma=\text { const. }
\end{gathered}
$$

Let $R_{0}$ and $Z_{0}$ will be the initial radius of the plume at $z=0$ and the initial height of the plume in $z$-direction, respectively. It is also used in [3] the mass $M_{P}$ of the plume and its initial energy $E_{P}$ as the input data. We will use the following values as the reference quantities: $l_{*}=R_{0}, v_{*}=\sqrt{(5 \gamma-3) E_{P} / M_{P}}$, $t_{*}=l_{*} / v_{*}, \rho_{*}=M_{P} /\left(R_{0}^{2} Z_{0}\right), p_{*}=\rho_{*} v_{*}^{2}$. It is assumed that the system (136)-(139) is written in the non-dimensional variables, since the dimensionless form of the gas dynamic equations coincides with (136)-(139), up to notations. The pseudo-analytic solution to (136)-139) can be written in the following form [3]:

$$
\begin{gathered}
\rho(r, z, t)=I_{1}^{-1}(\gamma) \frac{\sigma}{\xi^{2} \eta}\left(1-\frac{r^{2}}{\xi^{2}}-\frac{z^{2}}{\eta^{2}}\right)^{1 /(\gamma-1)}, \xi=\xi(t), \eta=\eta(t), \\
p(r, z, t)=\frac{I_{2}^{-1}(\gamma)}{5 \gamma-3}\left(\frac{\sigma}{\xi^{2} \eta}\right)^{\gamma}\left(1-\frac{r^{2}}{\xi^{2}}-\frac{z^{2}}{\eta^{2}}\right)^{\gamma /(\gamma-1)}, \quad \sigma=\frac{Z_{0}}{R_{0}}, \\
v_{r}(r, z, t)=r \frac{\dot{\xi}}{\xi}, v_{z}(r, z, t)=z \frac{\dot{\eta}}{\eta}, \quad \dot{\xi} \equiv \frac{d \xi}{d t}, \dot{\eta} \equiv \frac{d \eta}{d t}
\end{gathered}
$$




$$
\xi \ddot{\xi}=\eta \ddot{\eta}=\left(\frac{\sigma}{\xi^{2} \eta}\right)^{\gamma-1}, \quad \xi(0)=1, \eta(0)=\sigma, \dot{\xi}(0)=\dot{\eta}(0)=0,
$$

where

$$
\begin{gathered}
I_{1}(\gamma)=\frac{\pi^{3 / 2}}{2} \Gamma\left(\frac{\gamma}{\gamma-1}\right) / \Gamma\left(\frac{\gamma}{\gamma-1}+\frac{3}{2}\right), \\
I_{2}(\gamma)=\frac{\pi^{3 / 2}}{2(\gamma-1)} \Gamma\left(\frac{\gamma}{\gamma-1}+1\right) / \Gamma\left(\frac{\gamma}{\gamma-1}+\frac{5}{2}\right),
\end{gathered}
$$

$\Gamma(\cdot)$ is the Gamma-function. Out of the plume, i.e. provided $(r / \xi)^{2}+(z / \eta)^{2}$ $>1$, the values of density $(\rho)$ and pressure $(p)$ as well as the components of velocity $\left(v_{r}, v_{z}\right)$ are equal to zero.

Thus, the problem, (136)-(139), is reduced to the system of ordinary differential equations (ODEs) (144). The system of ODEs, (144), is solved numerically by the Runge-Kutta method with the adaptive step-size control [46]. To check the accuracy of the calculations, the integral of energy [3] is used:

$$
\dot{\xi}^{2}+0.5 \dot{\eta}^{2}+\frac{1}{\gamma-1}\left(\frac{\sigma}{\xi^{2} \eta}\right)^{\gamma-1}=\frac{1}{\gamma-1} .
$$

Aiming to use the schemes COS2, (59), and MAC2, (64), for solving the system (136)-(139), we rewrite (136)-(139) to read

$$
\begin{gathered}
\frac{\partial \rho}{\partial t}+\frac{\partial\left(\rho v_{r}\right)}{\partial r}+\frac{\partial\left(\rho v_{z}\right)}{\partial z}=-\frac{\rho v_{r}}{r} \\
\frac{\partial}{\partial t}\left(\rho v_{r}\right)+\frac{\partial}{\partial r}\left[\rho\left(v_{r}\right)^{2}+p\right]+\frac{\partial}{\partial z}\left(\rho v_{z} v_{r}\right)=-\frac{\rho\left(v_{r}\right)^{2}}{r} \\
\frac{\partial}{\partial t}\left(\rho v_{z}\right)+\frac{\partial}{\partial z}\left[\rho\left(v_{z}\right)^{2}+p\right]+\frac{\partial}{\partial r}\left(\rho v_{z} v_{r}\right)=-\frac{\rho v_{z} v_{r}}{r} \\
\frac{\partial \rho E}{\partial t}+\frac{\partial}{\partial r}\left[v_{r}(\rho E+p)\right]+\frac{\partial}{\partial z}\left[v_{z}(\rho E+p)\right]=-\frac{v_{r}(\rho E+p)}{r} .
\end{gathered}
$$

The initial conditions are the following (in details, see (141)-(146) under $t=0)$ :

$$
v_{r}=v_{z}=0, \rho=I_{1}^{-1}(\gamma)\left(1-r^{2}-(z / \sigma)^{2}\right)^{1 /(\gamma-1)}, p / \rho^{\gamma}=\text { const } .
$$

The boundary conditions are established on the basis of the reflection concept 2. At $r=0$ we assume that the axis $z$ is a reflection line. It prohibits any normal flux of mass through the boundary $r=0$, i.e. $v_{r}=0$. Moreover, it is assumed that the pressure $(p)$, density $(\rho)$, and tangential velocity $\left(v_{z}\right)$ are even functions of normal distance to the axis $z$ while the normal velocity $\left(v_{r}\right)$ is an odd function of $r$. It is also assumed that the plane $z=0$ is a reflection surface, i.e. the pressure $(p)$, density $(\rho)$, and tangential velocity $\left(v_{r}\right)$ are even functions of normal distance above the target surface while the normal velocity $\left(v_{z}\right)$ is an odd function of $z$. 
By virtue of the operator-splitting method [6], [13, [19], 32] (see also LOS in [48), the system (148)-(151) may be approximated by the following chain of equations:

$$
\begin{gathered}
\frac{1}{4} \frac{\partial \check{\mathbf{u}}}{\partial t}+\frac{\partial}{\partial z} \check{\mathbf{f}}(\check{\mathbf{u}})=0, \quad t_{n}<t \leq t_{n+0.25},\left.\quad \check{\mathbf{u}}\right|_{t=t_{n}}=\hat{\mathbf{u}}^{n} \\
\frac{1}{4} \frac{\partial \overline{\mathbf{u}}}{\partial t}+\frac{\partial}{\partial r} \overline{\mathbf{f}}(\overline{\mathbf{u}})=0, t_{n+0.25}<t \leq t_{n+0.5},\left.\quad \overline{\mathbf{u}}\right|_{t=t_{n+0.25}}=\check{\mathbf{u}}^{n+0.25} \\
\frac{1}{2} \frac{\partial \hat{\mathbf{u}}}{\partial t}=-\frac{1}{r} \mathbf{q}(\hat{\mathbf{u}}), t_{n+0.5}<t \leq t_{n+1},\left.\quad \hat{\mathbf{u}}\right|_{t=t_{n+0.5}}=\overline{\mathbf{u}}^{n+0.5}
\end{gathered}
$$

where $\check{\mathbf{u}}=\left\{\check{\rho}, \check{\rho} \check{v}_{r}, \check{\rho} \check{v}_{z}, \check{\rho} \check{E}\right\}^{T}, \check{\mathbf{f}}(\check{\mathbf{u}})=\left\{\check{\rho} \check{v}_{z}, \check{\rho}_{z} \check{v}_{z}, \check{\rho}\left(\check{v}_{z}\right)^{2}+\check{p}, \check{v}_{z}(\check{\rho} \check{E}+\check{p})\right\}^{T}$, $\overline{\mathbf{u}}=\left\{\bar{\rho}, \bar{\rho} \bar{v}_{r}, \bar{\rho} \bar{v}_{z}, \bar{\rho} \bar{E}\right\}^{T}, \overline{\mathbf{f}}(\overline{\mathbf{u}})=\left\{\bar{\rho} \bar{v}_{r}, \bar{\rho}\left(\bar{v}_{r}\right)^{2}+\bar{p}, \bar{\rho} \bar{v}_{z} \bar{v}_{r}, \bar{v}_{r}(\bar{\rho} \bar{E}+\bar{p})\right\}^{T}$, $\hat{\mathbf{u}}=\left\{\hat{\rho}, \hat{\rho} \hat{v}_{r}, \hat{\rho} \hat{v}_{z}, \hat{\rho} \hat{E}\right\}^{T}, \mathbf{q}(\hat{\mathbf{u}})=\left\{\hat{\rho} \hat{v}_{r}, \hat{\rho}\left(\hat{v}_{r}\right)^{2}, \hat{\rho} \hat{v}_{z} \hat{v}_{r}, \hat{v}_{r}(\hat{\rho} \hat{E}+\hat{p})\right\}^{T}$.

We will use the schemes COS2, (59), and MAC2, (64), fo solving Eqs. (153)(154). To solve Eq. (155) it will be used the Runge-Kutta scheme (modified Euler method), (111). Let us note that every point on the axis $r=0$ is a singular point for Eq. (155). Assuming that all terms at left-hand side of Equation (148) are bounded values at a vicinity of $r=0$, we find that $v_{r} \rightarrow 0$ as $r \rightarrow 0$ and, hence, $\left.\mathbf{q}(\hat{\mathbf{u}})\right|_{r=0}=0$. Thus

$$
\lim _{r \rightarrow 0} \frac{\left.\mathbf{q}(\hat{\mathbf{u}})\right|_{r>0}}{r}=\lim _{r \rightarrow 0} \frac{\left.\mathbf{q}(\hat{\mathbf{u}})\right|_{r>0}-\left.\mathbf{q}(\hat{\mathbf{u}})\right|_{r=0}}{r}=\left.\frac{\partial \mathbf{q}(\hat{\mathbf{u}})}{\partial r}\right|_{r=0} .
$$

Thus, we have at $r=0$ :

$$
\frac{1}{2} \frac{\partial \hat{\mathbf{u}}}{\partial t}=-\frac{\partial \mathbf{q}(\hat{\mathbf{u}})}{\partial r}, t_{n+0.5}<t \leq t_{n+1},\left.\quad \hat{\mathbf{u}}\right|_{t=t_{n+0.5}}=\overline{\mathbf{u}}^{n+0.5} .
$$

Let $\Delta r, \Delta z$ denote the spatial increments and let $r_{i}=i \Delta r, z_{j}=j \Delta z$, $i, j=0,1,2, \ldots$. The Runge-Kutta scheme, for Eq. (155) can be written in the form:

$$
\begin{gathered}
\hat{\mathbf{v}}_{i, j}^{n+0.75}=\hat{\mathbf{v}}_{i, j}^{n+0.5}-\frac{\Delta t}{2 r_{i}} \mathbf{q}\left(\hat{\mathbf{v}}_{i, j}^{n+0.5}\right), \\
\hat{\mathbf{v}}_{i, j}^{n+1}=\hat{\mathbf{v}}_{i, j}^{n+0.5}-\frac{\Delta t}{r_{i}} \mathbf{q}\left(\hat{\mathbf{v}}_{i, j}^{n+0.75}\right), i=1,2, \ldots, j=0,1,2, \ldots,
\end{gathered}
$$

where $\hat{\mathbf{v}}_{i, j}^{n+\theta}=\left\{(\hat{\rho})_{i, j}^{n+\theta},\left(\hat{\rho} \hat{v}_{r}\right)_{i, j}^{n+\theta},\left(\hat{\rho} \hat{v}_{z}\right)_{i, j}^{n+\theta},(\hat{\rho} \hat{E})_{i, j}^{n+\theta}\right\}^{T}, \theta=0.5,0.75,1$. If $i=0$, then, in view of (157), the Runge-Kutta scheme is the following

$$
\hat{\mathbf{v}}_{0, j}^{n+0.75}=\hat{\mathbf{v}}_{0, j}^{n+0.5}-\left.\frac{\Delta t}{2} \frac{\partial \mathbf{q}}{\partial r}\right|_{r=0} ^{n+05}, \hat{\mathbf{v}}_{0, j}^{n+1}=\hat{\mathbf{v}}_{0, j}^{n+0.5}-\left.\Delta t \frac{\partial \mathbf{q}}{\partial r}\right|_{r=0} ^{n+0.75}, j \geq 0 .
$$

Notice, we are using, for the sake of convenience, the same notation for the components of, in general, different vectors $\hat{\mathbf{u}}$ and $\hat{\mathbf{v}}$. To derive the scheme at $i=0$, we will use the reflection concept [2]. We introduce a node $i=-1$, i.e., 
$r_{-1}=-\Delta r$. At this node, in view of the reflection concept, we have: $\hat{\mathbf{v}}_{-1, j}^{n+\theta}=$ $\left\{(\hat{\rho})_{1, j}^{n+\theta},-\left(\hat{\rho} \hat{v}_{r}\right)_{1, j}^{n+\theta},\left(\hat{\rho} \hat{v}_{z}\right)_{1, j}^{n+\theta},(\hat{\rho} \hat{E})_{1, j}^{n+\theta}\right\}^{T}, \theta=0.5,0.75, j=0,1,2, \ldots$ Then, using central differencing, we obtain, by virtue of (159), the following difference scheme for Eq. (157):

$$
\begin{gathered}
\hat{\mathbf{v}}_{0, j}^{n+0.75}=\hat{\mathbf{v}}_{0, j}^{n+0.5}-\frac{\Delta t}{4 \Delta r}\left[\mathbf{q}\left(\hat{\mathbf{v}}_{1, j}^{n+0.5}\right)-\mathbf{q}\left(\hat{\mathbf{v}}_{-1, j}^{n+0.5}\right)\right], \\
\hat{\mathbf{v}}_{0, j}^{n+1}=\hat{\mathbf{v}}_{0, j}^{n+0.5}-\frac{\Delta t}{2 \Delta r}\left[\mathbf{q}\left(\hat{\mathbf{v}}_{1, j}^{n+0.75}\right)-\mathbf{q}\left(\hat{\mathbf{v}}_{-1, j}^{n+0.75}\right)\right], \quad j=0,1,2, \ldots .
\end{gathered}
$$

The equations (136)-(139) are integrated up to $t=1$ with $\sigma \equiv Z_{0} / R_{0}=$ 0.1. It is assumed for the schemes COS2, (59), and MAC2, (64), that the monotonicity parameter $\aleph=0.2$ and the parameter $\varkappa=1$. In view of the stability conditions, (67) we take $C r=0.95$ for the scheme COS2. Following to [23, we take $C r=0.475$ for the scheme MAC2, in contrast to the sufficient (but not necessary) stability conditions (86). It is assumed, in the case of scheme COS2, that the spatial increments are the following: $\Delta r=10^{-3}, \Delta z=5 \times 10^{-4}$ if $0<t \leq 0.1 ; \Delta r=2 \times 10^{-3}, \Delta z=10^{-3}$ if $0.1<t \leq 0.4 ; \Delta r=2.0 \times 10^{-3}$, $\Delta z=2.0 \times 10^{-3}$ if $0.4<t \leq 1$. Taking into account the fact that in the case of the staggered scheme COS2 the space increment is, actually, two times less than in the case of the nonstaggered scheme MAC2, we assume for the scheme MAC2: $\Delta r=5 \times 10^{-4}, \Delta z=2.5 \times 10^{-4}$ if $0<t \leq 0.1 ; \Delta r=10^{-3}, \Delta z=5 \times 10^{-4}$ if $0.1<t \leq 0.4 ; \Delta r=10^{-3}, \Delta z=10^{-3}$ if $0.4<t \leq 1$. The results of simulations as well as the analytical solution are depicted in Figures 7, 8, 9, 10,

We observe (Figures 7, 8, 9, 10) that the numerical results obtained via the schemes COS2 and MAC2 are practically coincide. We also observe that the relatively large deviations between numerical and analytical solutions are occurred in the area of the relatively large second derivatives (i.e. their absolute values) of the primitive variables over the spatial coordinates. Hence, these relatively large deviations may be occurred in the area of small values of the variables in the vicinity of the front (see Figures 7, 8, 9] 10) as well as in the area of relatively large values as well (see Figure 8).

\section{References}

[1] I. Ahmad., M. Berzins, MOL solvers for hyperbolic PDEs with source terms, Mathematics and Computers in Simulation 56 (2001) 115-125

[2] D. A. Anderson, J. C. Tannehill and R. H. Pletcher, Computational Fluid Mechanics and Heat Transfer, Hemisphere Publishing Corporation, New York, 1984.

[3] S. I. Anisimov, D. Bäuerle, B. S. Luk'yanchuk, Gas dynamics and film profiles in pulsed-laser deposition of materials, Phys. Rev. B 48 (16) (1993) 12 076-12 081. 

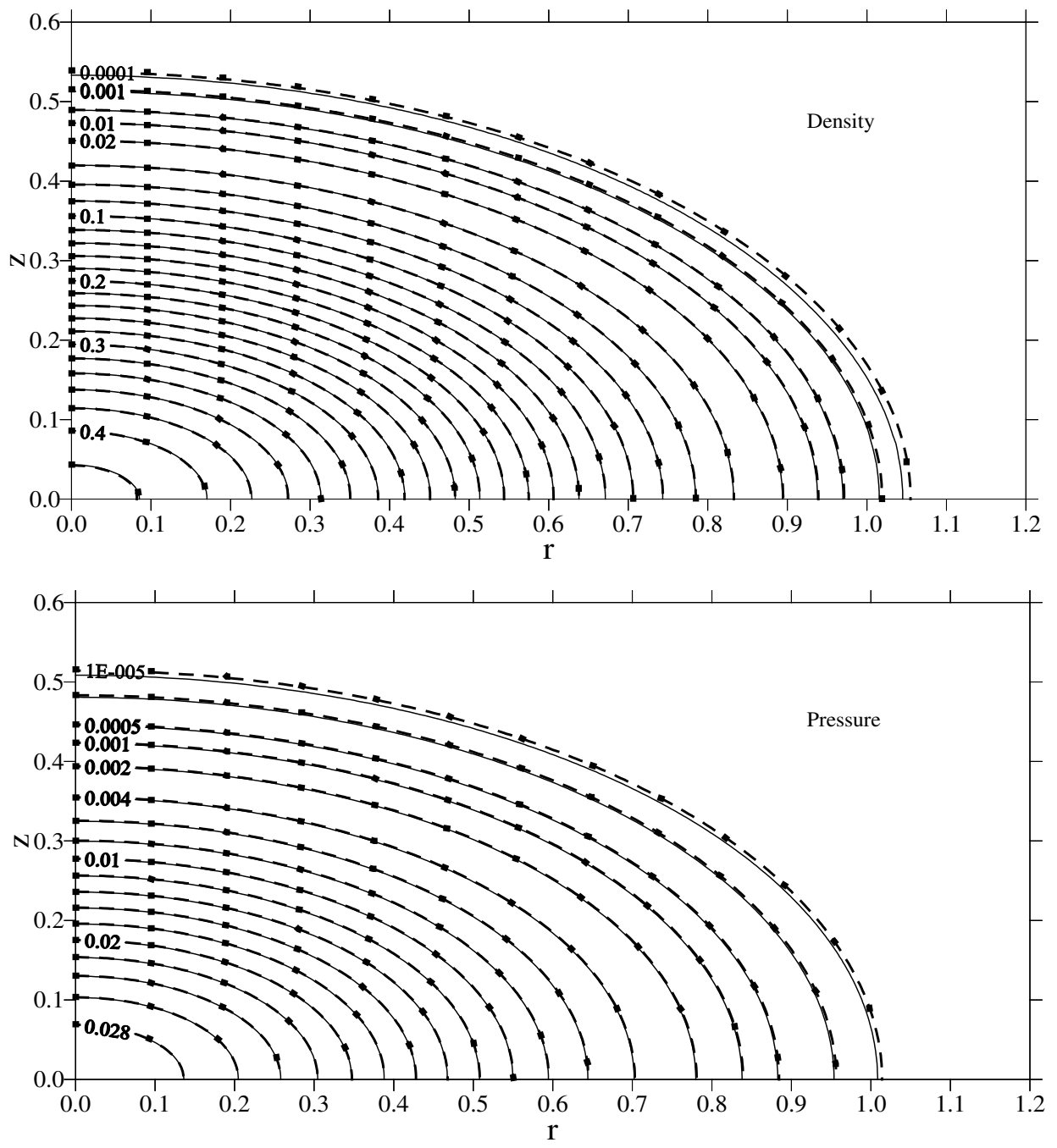

Figure 7: Anisimov's problem, density and pressure distribution. COS2 $(\mathrm{Cr}$ $=0.95)$ and MAC2 $(C r=0.475)$ schemes versus the analytical solution. $\sigma \equiv$ $Z_{0} / R_{0}=0.1$, time $t=0.4$. The monotonicity parameter $\aleph=0.2$ and the parameter $\varkappa=1$. Dashed lines: numerical solution (COS2); Square dotted lines: numerical solution (MAC2); Solid lines: analytical solution. 

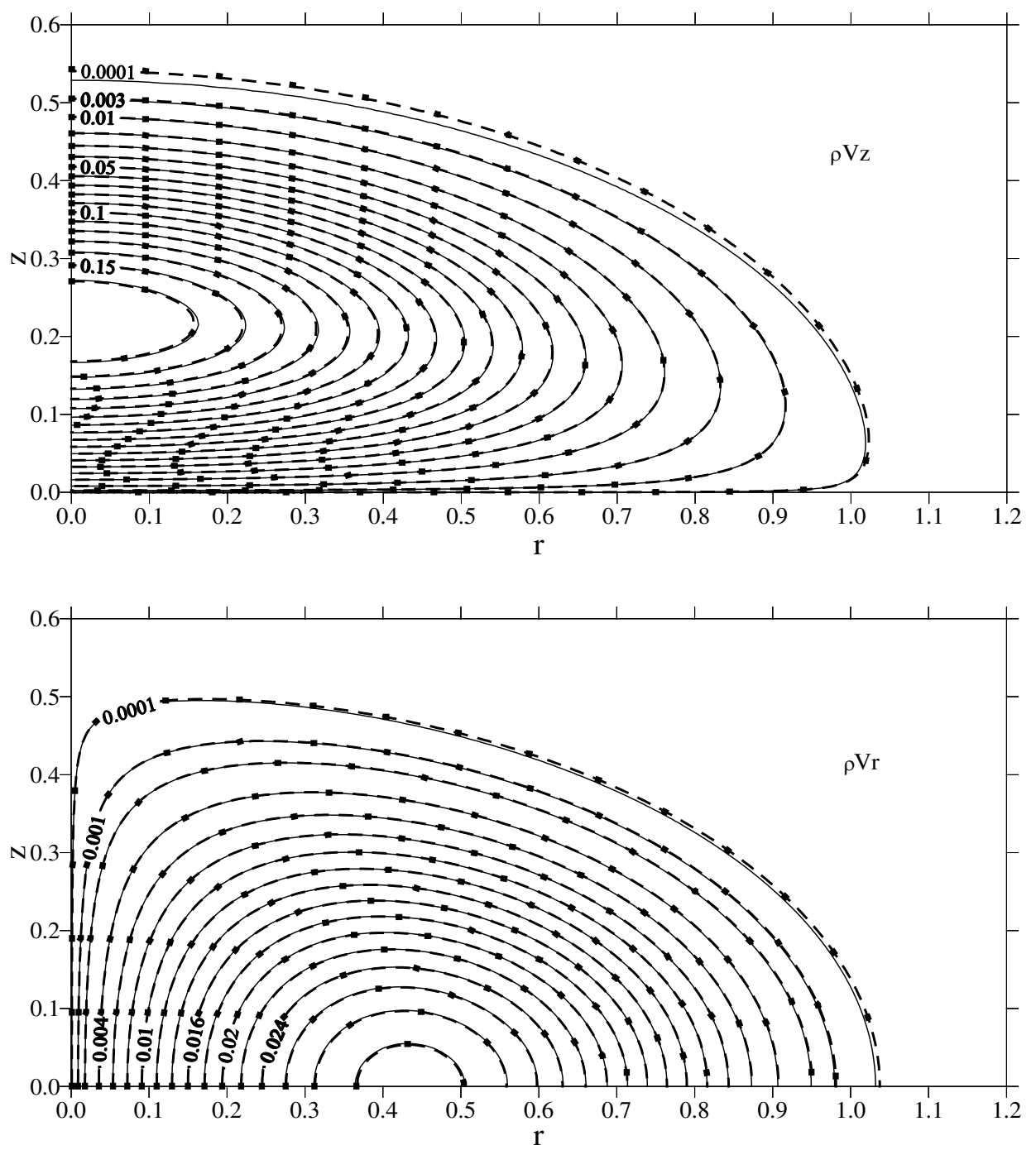

Figure 8: Anisimov's problem, momenta $\left(\rho V_{z}\right.$ and $\left.\rho V_{r}\right)$ distribution. COS2 $(C r=0.95)$ and MAC2 $(C r=0.475)$ schemes versus the analytical solution. $\sigma \equiv Z_{0} / R_{0}=0.1$, time $t=0.4$. The monotonicity parameter $\aleph=0.2$ and the parameter $\varkappa=1$. Dashed lines: numerical solution (COS2); Square dotted lines: numerical solution (MAC2); Solid lines: analytical solution. 


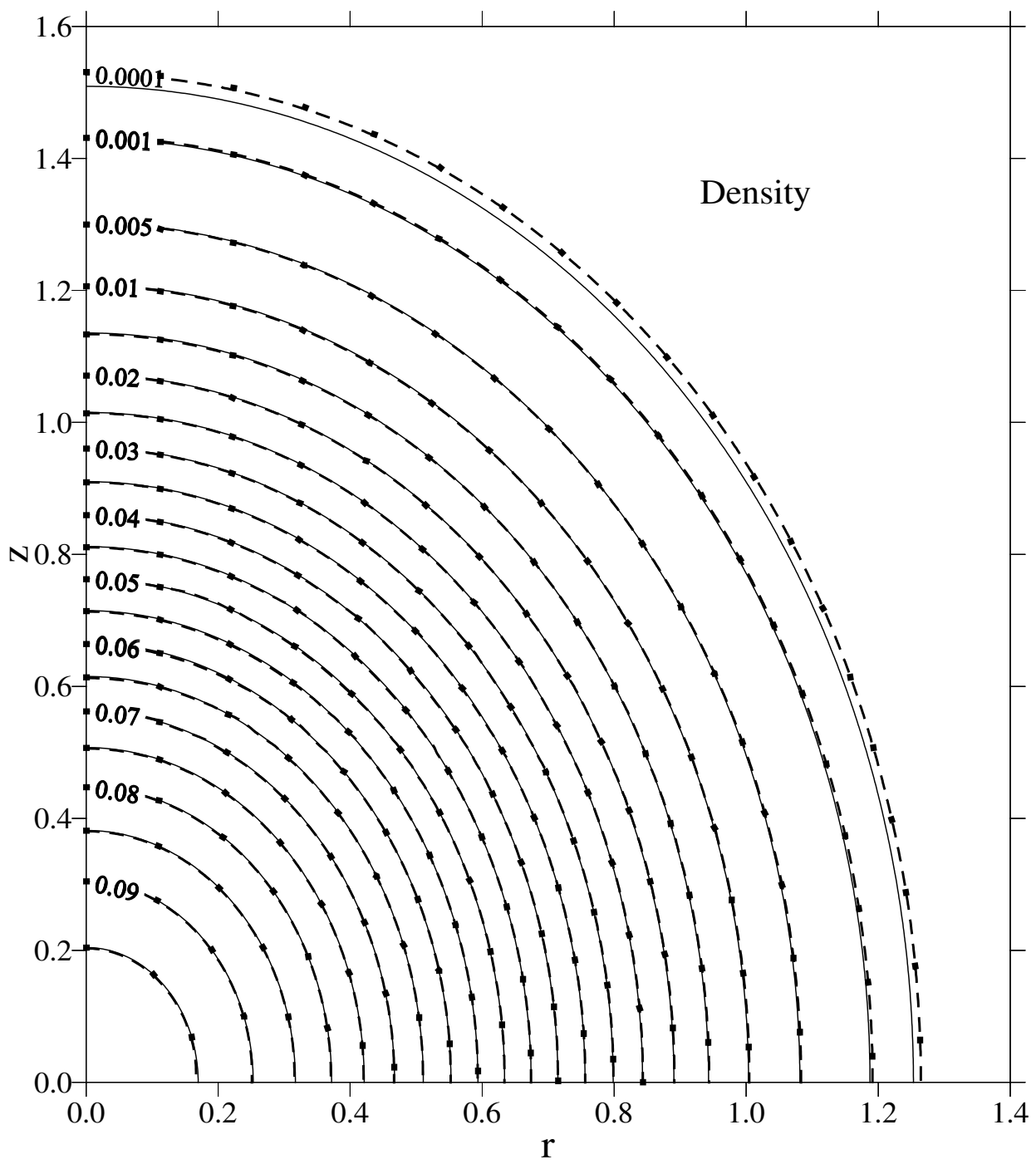

Figure 9: Anisimov's problem, density distribution. COS2 $(\mathrm{Cr}=0.95)$ and MAC2 $(C r=0.475)$ schemes versus the analytical solution. $\sigma \equiv Z_{0} / R_{0}=0.1$, time $t=1$. The monotonicity parameter $\aleph=0.2$ and the parameter $\varkappa=$ 1. Dashed lines: numerical solution (COS2); Square dotted lines: numerical solution (MAC2); Solid lines: analytical solution. 


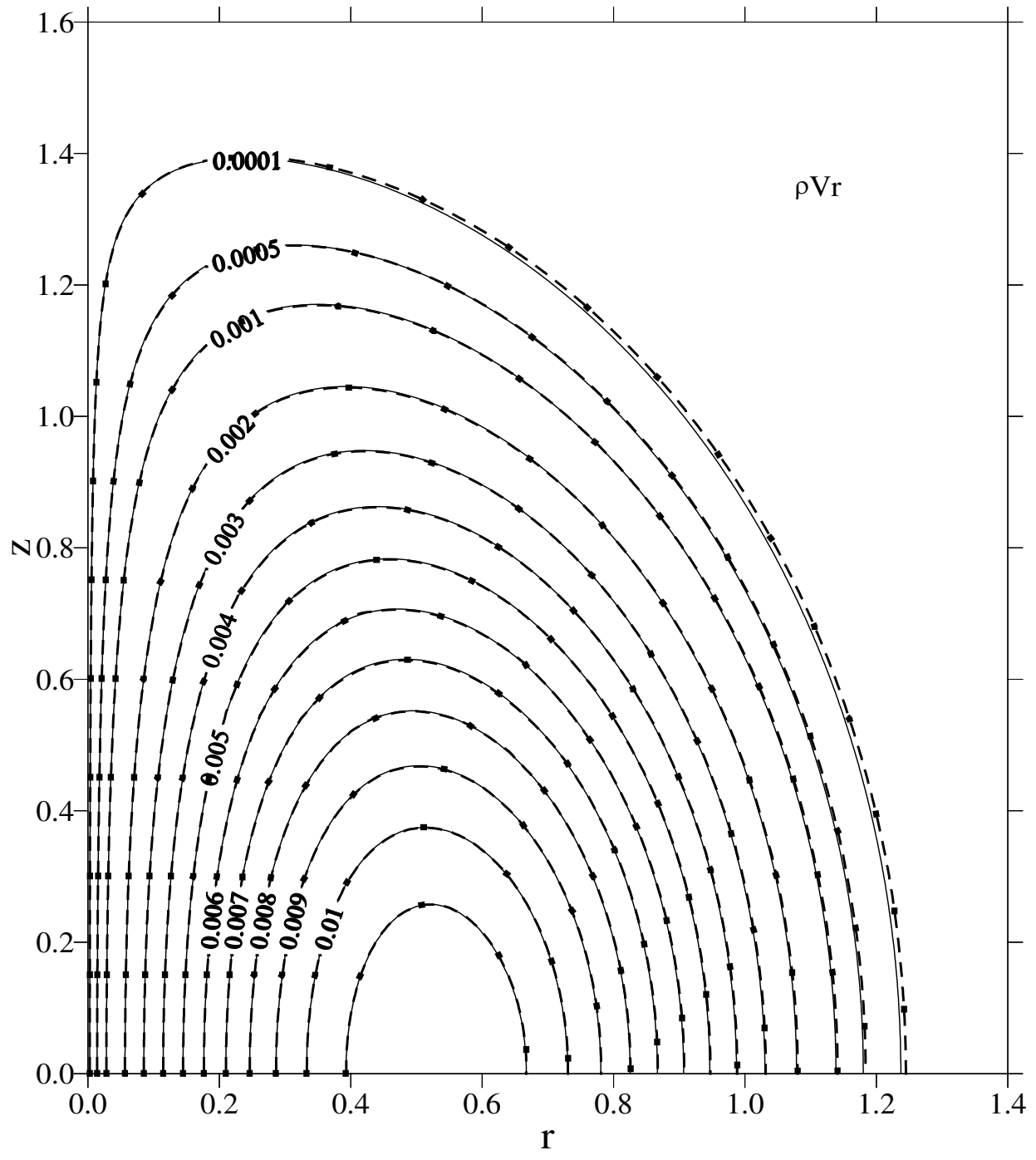

Figure 10: Anisimov's problem, momentum, $\rho V_{r}$, distribution. COS2 $(\mathrm{Cr}=$ $0.95)$ and $\mathrm{MAC} 2(\mathrm{Cr}=0.475)$ schemes versus the analytical solution. $\sigma \equiv$ $Z_{0} / R_{0}=0.1$, time $t=1$. The monotonicity parameter $\aleph=0.2$ and the parameter $\varkappa=1$. Dashed lines: numerical solution (COS2); Square dotted lines: numerical solution (MAC2); Solid lines: analytical solution. 
[4] Mark A. Aves, David F. Griffiths, and Desmond J. Higham, Runge-Kutta solutions of a hyperbolic conservation law with source term, SIAM J. Sci. Comput., Vol. 22, No. 1, pp. 20-38 (2000)

[5] Angel Balaguer and Carlos Conde, Fourth-order non-oscillatory upwind and central schemes for hyperbolic conservation laws, SIAM J. Numer. Anal. , Vol. 43, No. 2, pp. 455-473, (2005)

[6] François Bereux, Lionel Sainsaulieu, A roe-type Riemann solver for hyperbolic systems with relaxation based on time-dependent wave decomposition, Numer. Math. 77: 143-185 (1997).

[7] V. S. Borisov and M Mond, On stability, monotonicity, and construction of difference schemes I: Theory, SIAM J. Sci. Comput., 32 (2010), pp. 27652792 .

[8] V. S. Borisov and M Mond, On stability, monotonicity, and construction of difference schemes II: Applications, SIAM J. Sci. Comput., 32 (2010), pp. 2793-2819.

[9] V. S. Borisov and S. Sorek, - On monotonicity of difference schemes for computational physics, SIAM J. Sci. Comput., Vol. 25, No. 5 (2004), pp. $1557-1584$.

[10] V. S. Borisov, On discrete maximum principles for linear equation systems and monotonicity of difference schemes, SIAM J. Matrix Anal. Appl., Vol. 24, No. 4 (2003), pp. 1110-1135.

[11] Victor I. Burenkov, Sobolev Spaces on Domains, B. G. Teubner, Leipzig, 1998.

[12] Russel E. Caflisch, Shi Jin, and Giovanni Russo, Uniformly accurate schemes for hyperbolic systems with relaxation, SIAM J. Numer. Anal., Vol. 34, No. 1 (1997), pp. 246-281.

[13] Tao Du, Jing Shi, Zi-Niu Wu, Mixed analytical/numerical method for flow equations with a source term, Computers \& Fluids 32 (2003) 659-690.

[14] F.N. Fritsch and R.E. Carlson, Monotone piecewise cubic interpolation, SIAM J. Numer. Anal. 17, No. 2, 238-246 (1980).

[15] V. G. Ganzha and E. V. Vorozhtsov, Numerical Solutions for Partial Differential Equations, CRC Press, New York, 1996.

[16] V. G. Ganzha and E. V. Vorozhtsov, Computer-aided analysis of difference schemes for partial differential equations, John Wiley \& Sons, New York, 1996.

[17] M. I. Gil', Difference Equations in Normed Spaces, Stability and Oscillations, Elsevier, Amsterdam, 2007. 
[18] Edwige Godlewski and Pierre-Arnaud Raviart, Numerical Approximation of Hyperbolic Systems of Conservation Laws, Springer-Verlag, New York, 1996.

[19] L. Gosse, A Well-Balanced Flux-Vector Splitting Scheme Designed for Hyperbolic Systems of Conservation Laws with Source Terms, Computers and Mathematics with Applications 39 (2000) 135-159

[20] David F. Griffiths, Desmond J. Higham, Numerical Methods for Ordinary Differential Systems: Initial Value Problems (Springer Undergraduate Mathematics Series), Springer-Verlag, London, 2010.

[21] Heinonen Juha, Lectures on Lipschitz analysis, Rep. Univ. Jyväskylä Dept. Math. Stat. 100 (2005), 1-77.

[22] Roger A. Horn and Charles R. Johnson, Topics in Matrix Analysis, Cambridge Univ. Press, 1991.

[23] G.-S. Jiang, D. Levy, C.-T. Lin, S. Osher, E. Tadmor, High-Resolution Nonoscillatory Central Schemes with Nonstaggered Grids for Hyperbolic Conservation Laws, SIAM Journal on Numerical Analysis, Vol. 35, No. 6. (Dec., 1998), pp. 2147-2168.

[24] Shi Jin, Runge-Kutta Methods for Hyperbolic Conservation Laws with Stiff Relaxation Terms, J. Comp. Phys. 122 (1995), 51-67.

[25] Shi Jin, Lorenzo Pareschi, and Gioseppe Toscani, Uniformly accurate diffusive schemes for multiscale transport equations, SIAM J. Numer. Anal. Vol. 38, No. 3 (2000), pp. 913-936.

[26] David Kahaner, Cleve Moler, and Stephen Nash, Numerical methods and software, Prentice-Hall, New Jersey, 1989.

[27] L.M. Kocić and G.V. Milovanović, Shape Preserving Approximations by Polynomials and Splines, Computers Math. Applic. Vol. 33, No. 11, pp. 59-97, 1997.

[28] Kolmogorov A. N., Fomin S. V. Introductory Real Analysis, Prentice Hall, USA, 1970.

[29] Alexander Kurganov and Eitan Tadmor, New High-Resolution Central Schemes for Nonlinear Conservation Laws and Convection-Diffusion Equations, Journal of Computational Physics 160, 241-282 (2000)

[30] Alexander Kurganov and Doron Levy, A third-order semidiscrete central scheme for conservation laws and convection-diffusion equations, SIAM J. Sci. Comput., Vol. 22, No. 4 (2000), pp. 1461-1488

[31] Giovanni Leoni, A first course in Sobolev spaces, American Mathematical Society, Providence, Rhode Island, 2009. 
[32] Randall J. LeVeque, Finite volume methods for hyperbolic problems, Cambridge University Press, Cambridge, 2002.

[33] Xu-Dong Liu and Eitan Tadmor, Third order nonoscillatory central scheme for hyperbolic conservation laws, Numer. Math. (1998) 79: 397-425.

[34] L. A. Monthé, A study of splitting scheme for hyperbolic conservation laws, Journal of Computational and Applied Mathematics 137 (2001) 1-12.

[35] K. W. Morton, Numerical Solution of Convection-Diffusion Problems, Chapman \& Hall, London, 1996.

[36] K. W. Morton, Discretization of unsteady hyperbolic conservation laws, SIAM J. Numer. Anal., Vol. 39, No. 5 (2001), pp. 1556-1597.

[37] Giovanni Naldi and Lorenzo Pareschi, Numerical schemes for hyperbolic systems of conservation laws with stiff diffusive relaxation, SIAM J. Numer Anal., Vol. 37, No. 4 (2000), pp. 1246-1270.

[38] Greg F. Naterer and Jose A. Camberos, Entropy Based Design and Analysis of Fluids Engineering Systems, ), Taylor and Francis Group, Boca Raton, USA, 2008.

[39] Haim Nessyahu and Eitan Tadmor, Non-oscillatory Central Differencing for Hyperbolic Conservation Laws, Journal of Computational Physics, Vol. 87, No 2., April 1990, pp. 408-463.

[40] J. M. Ortega and W. C. Rheinboldt, Iterative solution of nonlinear equations in several variables, Academic Press, New York, London, 1970.

[41] Lorenzo Pareschi, Central differencing based numerical schemes for hyperbolic conservation laws with relaxation terms, SIAM J. Numer. Anal., Vol. 39, No. 4 (2001), pp. 1395-1417.

[42] Lorenzo Pareschi and Giovanni Russo, Implicit-Explicit Runge-Kutta Schemes and Applications to Hyperbolic Systems with Relaxation, Journal of Scientific Computing, Vol. 25, Nos. 1/2, November 2005, pp. 129-155.

[43] Lorenzo Pareschi, Gabriella Puppo, and Giovanni Russo, Central RungeKutta Schemes for conservation laws, SIAM J. Sci. Comput., Vol. 26, No. 3 (2005), pp. 979-999.

[44] Richard B. Pember, Numerical methods for hyperbolic conservation laws with stiff relaxation I. Spurious solutions, SIAM J. Appl. Math., Vol. 53, No. 5, pp. 1293-1330, October 1993.

[45] Richard B. Pember, Numerical methods for hyperbolic conservation laws with stiff relaxation II. Higher-order Godunov methods, SlAM J. Sci. Comput., Vol. 14, No. 4, pp. 826-859, July 1993. 
[46] William H. Press, Brian P. Flannery, Saul A. Teukolsky, William T. Vetterling, Numerical Recipes in C, The Art of Scientific Computing, Cambridge University Press, New York, 1988.

[47] R. D. Richtmyer and K. W. Morton, Difference Methods for Initial-Value Problems, 2nd edn, Wiley-Interscience, New York, 1967.

[48] A. A. Samarskii, The theory of difference schemes, Marcel Dekker, New York, 2001.

[49] A. A. Samarskiy and A.V. Gulin, Stability of Finite Difference Schemes, Nauka, Moscow, 1973 (in Russian).

[50] Susana Serna and Antonio Marquina, Capturing shock waves in inelastic granular gases, Journal of Computational Physics 209 (2004) 787-795.

[51] Dmitry Strunin, Nonlinear instability in generalized nonlinear phase diffusion equation, Progress of Theoretical Physics Supplement No. 150, pp. 444-448, 2003

[52] Huijiang Zhao and Shaoqiang Tang, Nonlinear Stability and Optimal Decay Rate for a Multidimensional Generalized Kuramoto]Sivashinsky System, Journal of Mathematical Analysis and Applications 246, 423-445, 2000 